\title{
iTRAQ-based proteome profile analysis of superior and inferior Spikelets at early grain filling stage in japonica Rice
}

\author{
Cuicui You ${ }^{1,2}$, Lin Chen ${ }^{2}$, Haibing He ${ }^{1}$, Liquan $\mathrm{Wu}^{1 *}$, Shaohua Wang ${ }^{2,3^{*}}$, Yanfeng Ding ${ }^{2,3}$ and Chuanxi Ma ${ }^{1}$
}

\begin{abstract}
Background: Large-panicle rice varieties often fail to achieve their yield potential due to poor grain filling of lateflowering inferior spikelets (IS). The physiological and molecular mechanisms of poor IS grain filling, and whether an increase in assimilate supply could regulate protein abundance and consequently improve IS grain filling for japonica rice with large panicles is still partially understood.

Results: A field experiment was performed with two spikelet removal treatments at anthesis in the large-panicle japonica rice line W1844, including removal of the top $1 / 3$ of spikelets (T1) and removal of the top 2/3 of spikelets (T2), with no spikelet removal as a control (TO). The size, weight, setting rate, and grain filling rate of IS were significantly increased after spikelet removing. The biological functions of the differentially expressed proteins (DEPs) between superior and inferior spikelets as well as the response of IS to the removal of superior spikelets (SS) were investigated by using iTRAQ at 10 days post anthesis. A total of 159, 87, and 28 DEPs were identified from group A (T0-SS/TO-IS), group B (TO-SS/T2-IS), and group C (T2-IS/TO-IS), respectively. Among these, 104, 63, and 22 proteins were up-regulated, and 55,24 , and 6 proteins were down-regulated, respectively. Approximately half of these DEPs were involved in carbohydrate metabolism (sucrose-to-starch metabolism and energy metabolism) and protein metabolism (protein synthesis, folding, degradation, and storage).

Conclusions: Reduced endosperm cell division and decreased activities of key enzymes associated with sucrose-starch metabolism and nitrogen metabolism are mainly attributed to the poor sink strength of IS. In addition, due to weakened photosynthesis and respiration, IS are unable to obtain a timely supply of materials and energy after fertilization, which might be resulted in the stagnation of IS development. Finally, an increased abundance of 14-3-3 protein in IS could be involved in the inhibition of starch synthesis. The removal of SS contributed to transfer of assimilates to IS and enhanced enzymatic activities of carbon metabolism (sucrose synthase, starch branching enzyme, soluble starch synthase, and pullulanase) and nitrogen metabolism (aspartate amino transferase and alanine amino transferase), promoting starch and protein synthesis in IS. In addition, improvements in energy metabolism (greater abundance of pyrophosphate-fructose 6-phosphate 1-phosphotransferase) might be played a vital role in inducing the initiation of grain filling. These results collectively demonstrate that carbohydrate supply is the main cause of poor IS grain filling.
\end{abstract}

Keywords: Rice, Removal of superior spikelets, Inferior spikelets, Grain filling, iTRAQ, Proteome

\footnotetext{
* Correspondence: wlq-001@163.com; wangsh@njau.edu.cn

${ }^{1}$ College of Agronomy, Anhui Agricultural University, Hefei 230036, People's

Republic of China

${ }^{2}$ College of Agronomy, Nanjing Agricultural University/Key Laboratory of

Crop Physiology Ecology and Production Management, Ministry of

Agriculture, Nanjing 210095, People's Republic of China

Full list of author information is available at the end of the article
} 


\section{Background}

Rice is a major staple food crop worldwide, and its consumption is increasing as the world's population grows. Improving the output per unit area is therefore necessary for producing more rice on a limited land area [1]. Many efforts have been made to reach this target by breeders, who have attempted to expand the sink capacity by increasing the number of spikelets per panicle, creating extra-heavy panicle types or large-panicle rice varieties [2]. These cultivars with larger sink capacities, however, generally do not produce the expected yield due to the low seed setting rate and grain weight of inferior spikelets (IS) [3, 4]. Within the rice panicle, spikelets are grouped into superior spikelets (SS) and IS according to their location on the branch and the time of flowering [5]. Generally, SS are located on upper primary branches, and they flower earlier, fill more quickly, and produce larger and heavier grains. The IS are located on the lower secondary branches, and they flower later, fill more slowly, and produce smaller grains [6]. Therefore, improving IS grain filling is important for achieving a high yield potential of large-panicle rice varieties.

There are many explanations that may account for poor IS grain filling, including reduced activities of the enzymes involved in sucrose-to-starch conversion $[7,8]$, hormone imbalances [4], assimilate transportation obstacles $[3,9]$, and the differential expression of genes associated with cell growth and signal transduction [10]. However, whether the assimilate supply is a crucial factor for poor IS grain filling remains controversy $[3,6,11]$. In order to clarify this question, previous studies have normally used leaf- and flowerthinning methods to regulate the source-sink balance $[12,13]$. For example, $\mathrm{Xu}$ et al. [14] found that IS grain weight and grain setting rate were significantly increased following removal of SS. However, Kato [15] reported that IS filling was not significantly improved by SS removal. Our previous study showed that SS removal could force assimilate transport to IS, promoting IS grain filling, and the possible physiological mechanisms underlying this process have been discussed [16]. However, rice grain filling is a highly complex biological process, and previous studies have primarily focused on the relationship between spikelet removal and grain weight or the underlying physiological mechanisms. Thus, the effect of SS removal on protein abundances in IS and how these interact with IS grain filling remains unclear.

In recent years, proteomics has become an essential technique for revealing the mechanisms of poor IS grain filling. Proteomics contributes to a greater understanding of complex biological systems as it allows for the simultaneous analysis of changes in multiple proteins
[17]. There have been numerous studies that have attempted to resolving the problem of poor IS grain filling by reporting differences in protein abundance between SS and IS. Zhang et al. [18] employed twodimensional gel electrophoresis (2-DE)-based comparative proteomic and phosphoproteomic analyses to explore differentially expressed proteins in IS following spraying with abscisic acid (ABA); a total of 111 differentially expressed proteins (DEPs) were found to be associated with defense response, carbohydrate, protein, amino acid, energy, secondary metabolism, cell development, and photosynthesis, demonstrating that IS grain filling was improved by ABA through proteins and phosphoproteins that participate in carbon, nitrogen, and energy metabolisms. Furthermore, Zhang et al. [19] reported that the 14-3-3 protein plays an important role in the signaling networks of IS development, especially in developmental stagnancy. Chen et al. [20] also compared differential protein expression between SS and IS using the 2-DE method and found that the dramatic down-regulation of functional proteins related to photosynthesis, carbohydrate and energy metabolism, amino acids metabolism, and defense responses was the main cause of poor IS grain filling. In addition, they found that post-anthesis alternate wetting and moderate soil drying could improve grain filling by regulating protein expression, especially in IS. Although 2-DE could separate thousands of different proteins and provide visual information of the proteome including distinct protein isoforms resulting from changes in Mr. (relative molecular mass) and pI (isoelectric point), it is not suitable for detection of low-abundance proteins and more accurate quantification. Isobaric tags for relative and absolute quantitation (iTRAQ) is a mass spectrometry-based quantitative approach that has become prevalent in developmental grain proteomics, as it simultaneously identifies and quantifies proteins from multiple samples with high coverage [21]. It has been reported that lower sink strength and smaller sink sizes result in reduced decomposition, conversion of photoassimilate, and slower cell division in hybrid rice [22]. However, previous studies were only made on hybrid rice or an indica varieties, and little proteomic information has documented using iTRAQ regarding SS and IS and the response of IS to SS removal in homozygous japonica rice.

This study investigated whether an increase in assimilate supply could regulate protein abundance and consequently improve IS grain filling for japonica rice with large panicles. Transfer of assimilates toward IS was forced by removal of SS, and we examined subsequent changes in grain weight, seed setting rate, and grain filling rate of IS during the grain filling period. Additionally, iTRAQ technology was used to identify DEPs between SS and IS under different treatments and their 
biological functions, and then we analyzed the relationship between these proteins and grain development to reveal the underlying causes of differences in grain filling between SS and IS as well as the response of IS to SS removal at proteomic level.

\section{Methods}

\section{Plant materials}

The experiment was conducted in 2015 at the Danyang Experimental Base of the Nanjing Agricultural University, Jiangsu Province, China ( $\left.31^{\circ} 54^{\prime} 31^{\prime \prime} \mathrm{N}, 119^{\circ} 28^{\prime} 21^{\prime \prime} \mathrm{E}\right)$ during the rice growing season. In order to analyze the mechanisms of poor IS grain filling at the molecular level, the experiment was conducted using the homozygous large-panicle japonica rice line W1844, which is an inbred line and not a hydrid or transgenetic line. Moreover, W1844 is the intermediate material of breeding, but its genetic characteristics have stabilized. The seeds of W1844 were provided by the professor jian-min wan of the State Key Laboratory of Rice Genetics and Germplasm Innovation, Nanjing Agricultural University, Jiangsu, China. W1844 has 265 grains per panicle and thus is typical of large-panicle rice varieties. Its plant height, panicle length, thousand-grain weight, and seed setting rate are $99.8 \mathrm{~cm}, 18.1 \mathrm{~cm}, 23.6 \mathrm{~g}$, and $92.1 \%$ respectively. Seeds were sown on May 28th, 2015, and seedlings were transplanted to the field on June 18th at a hill spacing of $13.3 \mathrm{~cm} \times 30 \mathrm{~cm}$. The trials were designed in randomized plots with three replicates, and each plot was $5 \mathrm{~m} \times 10 \mathrm{~m}$. The soil type was clay loam, and $280 \mathrm{~kg} \cdot \mathrm{ha}^{-1}$ nitrogen was applied during the growing season. Nitrogen fertilizer was converted into urea according to the nitrogen content and was applied according to the ratio of base fertilizer to panicle fertilizer (5:5). The base fertilizer was applied before seedling transplantation, and the panicle fertilizer was applied when the leaf-age remainder was 3.5. Cultivation and management measures were applied according to the technical requirements of the local high-yield field.

\section{Experimental design}

A total of 800 single stems (panicles) with similar growth patterns that flowered on the same day were labeled during heading-blooming stage. Once most labeled panicles had withdrawn from flag leaf sheath completely, two spikelet thinning treatments were performed: T0 was control treatment with no spikelet thinning, T1 plants had the upper $1 / 3$ of spikelets removed, and T2 plants had the upper $2 / 3$ of spikelets removed. Spikelet thinning involved removal of the primary branch. The primary branches of each panicle were equally divided into three parts: upper, middle, and lower. If the number of primary branches could not be divided equally, a number of spikelets equal to the integer of the average branch number was included in each of the upper and lower parts, and the remaining branches were included in the middle part. SS were considered to be the grains on the three primary branches on the upper part of the panicle, while medium spikelets (MS) were defined as the grains on the three primary branches in the middle part of the panicle, and IS were the grains on the three primary branches in the lower part.

\section{Sampling and measurement \\ Determination of the grain filling rate}

From anthesis to maturity, 50 tagged panicles from each plot were collected every 5 days. SS, MS, and IS samples were collected from the T0 group; MS and IS were collected from the T1 group, and only IS were collected from the T2 group. Two-fifths of the sampled grains were frozen in liquid nitrogen and stored at $-80{ }^{\circ} \mathrm{C}$ for protein extraction. The remaining grains were deactivated at $105{ }^{\circ} \mathrm{C}$ for $0.5 \mathrm{~h}$ and dried at $80{ }^{\circ} \mathrm{C}$ until they reached a constant weight. They were then weighed to determine grain dry weights. Richards's growth eq. [23] was used for grain filling process fitting and grain filling rate calculation:

$$
W=\frac{A}{\left(1+B e^{-k t}\right)^{1 / N}}
$$

The grain filling rate $(\mathrm{R})$ was calculated as the derivative of the Eq. (1)

$$
R=\frac{A k B e^{-k t}}{N\left(1+B e^{-k t}\right)^{(N+1) / N}}
$$

where $W$ is the grain weight $(\mathrm{mg}) ; A$ is the final grain weight (mg); $t$ is the time after anthesis (days); and $B, k$, and $N$ are coefficients established from the regression of the equation.

\section{Protein extraction}

Protein extraction was performed according to Isaacson et al. [24] with some modifications. About $0.1 \mathrm{~g}$ dehulled grains were homogenized with a pestle in a pre-cooled mortar containing ice-cold 10\% $(w / v)$ trichloroacetic acid in acetone. They were incubated at $-20{ }^{\circ} \mathrm{C}$ for $1 \mathrm{~h}$, followed by centrifugation at $15000 \mathrm{~g}$ for $15 \mathrm{~min}$ at $4{ }^{\circ} \mathrm{C}$ in a refrigerated high-speed centrifuge, after which the precipitate was collected. After vacuum drying, adding an equal volume of phenol saturated with Tris- $\mathrm{HCl}$ ( $\mathrm{pH} 7.5$ ), and centrifugation at $5000 \mathrm{~g}$ for $30 \mathrm{~min}$ at $4{ }^{\circ} \mathrm{C}$, we collected the upper phenolic phase. Five volumes of pre-cooled $0.1 \mathrm{M}$ ammonium acetate in methanol were added to collected phenol phase, followed by centrifugation at $10000 \mathrm{~g}$ for $10 \mathrm{~min}$ at $4{ }^{\circ} \mathrm{C}$, after which the precipitate was collected; this 
process was repeated three times. Protein concentration was determined by the BCA method [25].

\section{Protein digestion and ITRAQ labeling}

Protein digestion was performed according to the method of FASP [26]. Five volumes of cold acetone were added to $100 \mu \mathrm{g}$ protein from each sample and centrifuged at $12000 \mathrm{rpm}$ for $10 \mathrm{~min}$ at $4{ }^{\circ} \mathrm{C}$, collected the precipitate and dried by speed vacuum concentrator. $50 \mu \mathrm{L}$ dissolution buffer was added for dissolve protein precipitation, and added $4 \mu \mathrm{L}$ reducing reagent, incubated at $60{ }^{\circ} \mathrm{C}$ for $1 \mathrm{~h}$, then added $2 \mu \mathrm{L}$ cysteine-blocking reagent at room temperature for $10 \mathrm{~min}$. Clean the protein solution by using $10 \mathrm{KDa}$ ultrafiltration tube to centrifuge at $12000 \mathrm{rpm}$ for $20 \mathrm{~min}$, and discarded the solution at the bottom of the collection tube; $100 \mu \mathrm{L}$ dissolution buffer was added to the ultrafiltration tube, then centrifuged at $12000 \mathrm{rpm}$ for $15 \mathrm{~min}$, discarded the solution at the bottom of the collection tube and repeat this step three times. Replace a new collection tube, $50 \mu \mathrm{L}$ sequencinggrade trypsin $(50 \mathrm{ng} / \mu \mathrm{L})$ was placed into the ultrafiltration tube, incubated at $37{ }^{\circ} \mathrm{C}$ for $12 \mathrm{~h}$, and centrifuged at $12000 \mathrm{rpm}$ for $20 \mathrm{~min}$, then collected the peptides. Transfered the filter units to new collection tube and added $50 \mu \mathrm{L}$ dissolution buffer to centrifuge the tube again, and combined the two filter solution, which contained peptides. The peptides were dried in a centrifugal speed vacuum concentrator.

Two biological replicates were performed for each sample for iTRAQ analysis. The peptides of each sample were labeled using iTRAQ 8-plex kits according to the manufacturer's manual (AB SCIEX Inc., USA). Labelling was performed by adding one reagent vial, containing an isobaric tag, to $110 \mu \mathrm{g}$ of dried peptides for each sample. The labelling reaction proceeded for $3 \mathrm{~h}$ at room temperature after which all the samples were pooled before application of separation techniques and mass spectrometry analysis. The labelling scheme was as follows: Tags 113 and 117, T0-SS; Tags 114 and 118, T0-IS; Tags 115 and 119, T2-IS.

\section{Two dimensional liquid chromatography tandem mass spectrometry (2D-LC-MSMS) analysis}

After labeling, all samples were pooled and purified using a strong cation exchange chromatography (SCX) column by Agilent 1200 HPLC (Agilent). The HPLC column was purchased from Agilent, and its parameters were as follows: the Analytical Guard Column $4.6 \times 12.5 \mathrm{~mm}$ 5-Micron; Narrow-Bore $2.1 \times 150 \mathrm{~mm}$ $5 \mu \mathrm{m}$ with $215 \mathrm{~nm}$ and $280 \mathrm{~nm}$ UV detection. Separation was performed at $0.3 \mathrm{~mL} / \mathrm{min}$ using a nonlinear binary gradient. Collected the first peptides from 0 to 5 mins, then collected each peptides with 4.5 mins interval for the 6-45 $\mathrm{min}$, and for the last peptides from 46 to
50 mins, with a total of 10 peptides. Dried every peptides in a vacuum freezed dryer for LC-MSMS Analysis.

The dried peptides were re-suspended with NanoRPLC buffer A ( $0.1 \%$ formic acid, $2 \%$ acetonitrile). The online Nano-RPLC was employed on the Eksigent nanoLC-Ultra $^{\mathrm{sm}}$ 2D System (AB SCIEX). The samples were loaded on C18 NanoLC trap column $(100 \mu \mathrm{m} \times 3 \mathrm{~cm}$, C18, $3 \mu \mathrm{m}, 150 \AA$ ) and washed by Nano-RPLC Buffer A at $2 \mu \mathrm{L} / \mathrm{min}$ for $10 \mathrm{mins}$. An elution gradient of $5-35 \%$ acetonitrile $(0.1 \%$ formic acid) in 70 mins gradient was used on an analytical ChromXP C18 column $(75 \mu \mathrm{m} \times 15 \mathrm{~cm}, \mathrm{C} 18,3 \mu \mathrm{m} 120 \AA)$ with spray tip. The LC fractions were analyzed using a Triple TOF 5600 mass spectrometer.

Mass spectrometer data acquisition was performed with a Triple TOF 5600 System (AB SCIEX, USA) fitted with a Nanospray III source (AB SCIEX, USA) and a pulled quartz tip as the emitter (New Objectives, USA). Data were acquired using an ion spray voltage of $2.5 \mathrm{kV}$, curtain gas of $30 \mathrm{PSI}$, nebulizer gas of 5 PSI, and an interface heater temperature of $150{ }^{\circ} \mathrm{C}$. For information dependent acquisition (IDA), survey scans were acquired in $250 \mathrm{~ms}$ and as many as 35 product ion scans were collected if they exceeded a threshold of 150 counts per second (counts/s) with a $2^{+}$to $5^{+}$charge-state. The total cycle time was fixed to $2.5 \mathrm{~s}$. A rolling collision energy setting was applied to all precursor ions for collisioninduced dissociation (CID). Dynamic exclusion was set for $1 / 2$ of peak width (18 s), and the precursor was then refreshed off the exclusion list.

\section{Bioinformatics analysis}

The proteins were identified in two biological replicates using the iTRAQ technique. Data were processed with Protein Pilot Software v. 5.0 (AB SCIEX, USA) against Oryza sativa database of UniProt using the Paragon algorithm [27]. The experimental data from tandem mass spectrometry were matched against theoretical data for protein identification. The iTRAQ 8-plex was chosen for protein quantification with unique peptides during the search. According to the abundances of proteins and the results of comparison among groups, the screening criteria for authentic proteins was an FDR $\leq 1 \%$ and a unique peptide $\geq 1$. The screening criteria for DEPs was a fold change $>1.5$ or $<0.67$ and a $p$-value $<0.05$. The bioinformatics data analysis tool, OmicsBean, was used to analyze the obtained proteomics data (http://www.omicsbean.cn/), in which distributions in biological functions, cell component and molecular functions were assigned to each protein based on Gene Ontology (GO) categories. The Kyoto Encyclopedia of Genes and Genomes (KEGG) pathway analysis was performed in order to enrich high-level functions in the defined biological systems. 


\section{Assessment of panicle characteristics}

Approximately 90 tagged panicles from each treatment were harvested at maturity. The SS, MS, and IS were collected from T0 group, MS and IS were collected from T1 group, and IS were collected from T2 group. The samples were naturally dried, and the grain weight and seed setting rate were measured. The seed setting rate was determined using the method of Kobata et al. [28].

\section{Statistical analysis}

For all statistical analyses, at least three biological replicates were used for each treatment and control. Statistical analyses of the data were accomplished by the standard analysis of variance (ANOVA) and mean values were tested by least significant difference (LSD) at the 5\% level using SPSS16.0.

\section{Results}

\section{Grain weight and grain setting rate}

Grain weights and seed setting rates were significantly different among the SS, MS, and IS of W1844 under T0 treatment, while SS has the highest and IS has the lowest values (Table 1). Compared with T0 group, the grain weights and seed setting rates of MS and IS in T1 group, as well as those of the IS in T2 group were all increased, with the greatest improvement in seed setting under T2 treatment. Therefore, in the subsequent analyses, we focused on the effect of T2 treatment on IS grain filling. As shown in Table 1, the grain weight and seed setting rate of IS under T2 were the same as those of SS under T0, demonstrating that SS removal significantly improved IS grain weight and seed setting rate.

\section{Grain filling of SS and IS}

The dynamic changes in grain weight and grain filling rate in W1844 during the grain filling period were shown in Fig. 1. The IS grain weight was consistently lower than that of SS throughout the filling process, while under T2 treatment, the IS grain weight began to increase and reached the SS level at 30 days post anthesis (DPA) (Fig. 1-a). We found that the initial and maximum grain filling rates of IS were consistently lower than those of SS, and peak grain filling also appeared later in IS than that in SS (Fig. 1-b). Compared with T0 treatment, T2 treatment significantly increased the initial and maximum grain filling rates of IS. Moreover, the peak value of IS grain filling rate under T2 was higher and occurred 5 days earlier than that of IS in T0 group. Changes in grain weight and grain filling rate indicated that removal of SS significantly improved IS grain filling.

\section{Grain morphology of SS and IS}

Changes in the kernel development dynamics of SS and IS under different treatments are shown in Fig. 2. We observed that the SS first elongated and then widened after flowering, and SS grain size showed a rapid increase. However, the IS developed slowly during the early stage of grain filling (days 5-15), and its grain morphology changed greatly at 20 DPA. Compared to IS under T0, grain size and grain weight of IS under T2 treatment increased significantly at 10 DPA (Fig. 1-a), indicating that important changes occurred within the kernel during this time and affected the development of the IS. Some studies have shown that the physiological activities of grain are significantly positively correlated with grain filling at the beginning of the filling stage $[29,30]$. Therefore, the subsequent experiment studied protein expression in the grains under different treatments at 10 DPA.

\section{DEPs in SS and IS at 10 DPA under different treatments}

In order to further study the reason behind the grain filling difference between SS and IS, as well as molecular mechanism of IS response to SS removal, we used comparative proteomics to analyze protein expression in SS and IS. A total of 4631 proteins were identified in two biological replicates using the iTRAQ technique and were subjected to comparative analysis. Protein abundances that changed by more than 1.5-fold or less than 0.67 -fold were selected. Following this criterion, a total of 174 types of proteins were detected which showed that there were differentially abundant between SS and IS under different treatments at 10 DPA.

Table 2 lists these DEPs between SS and IS under different treatments, providing the accession numbers and names of these proteins according to the Uniprot database as well as their fold changes in abundance. The numbers of DEPs and their changes in abundance are listed in Fig. 3. As Fig. 3 shows, in the T0-SS/T0-IS comparison, 159 DEPs were identified, of which 104 proteins (65.4\%) were up-regulated and 55 proteins $(34.6 \%)$ were down-regulated; in the T0-SS/T2-IS comparison, 87

Table 1 Grain weight and seed setting rate under different treatments

\begin{tabular}{|c|c|c|c|c|c|c|}
\hline \multirow[t]{2}{*}{ Treatments } & \multicolumn{3}{|l|}{ T0 } & \multicolumn{2}{|l|}{$\mathrm{T} 1$} & \multirow{2}{*}{$\begin{array}{l}\text { T2 } \\
\text { Inferior }\end{array}$} \\
\hline & Superior & Medium & Inferior & Medium & Inferior & \\
\hline Grain weight (mg/grain) & $26.6 \pm 0.13 a$ & $23.2 \pm 0.53 c$ & $20.9 \pm 0.39 d$ & $26.2 \pm 0.23 b$ & $25.6 \pm 0.45 b$ & $28.2 \pm 0.12 \mathrm{a}$ \\
\hline Seed setting rate (\%) & $97.1 \pm 0.47 a$ & $93.5 \pm 0.39 b$ & $85.7 \pm 0.26 c$ & $96.8 \pm 0.26 a$ & $92.5 \pm 1.10 b$ & $96.6 \pm 0.76 a$ \\
\hline
\end{tabular}

T0: control treatment with no spikelet thinning; T1: the upper $1 / 3$ of spikelets were removed; T2: the upper $2 / 3$ of spikelets were removed. Values are means \pm S.D. of three replications. The different lowercase letters labeled after the data from the same character indicate significant differences at the 0.05 level 

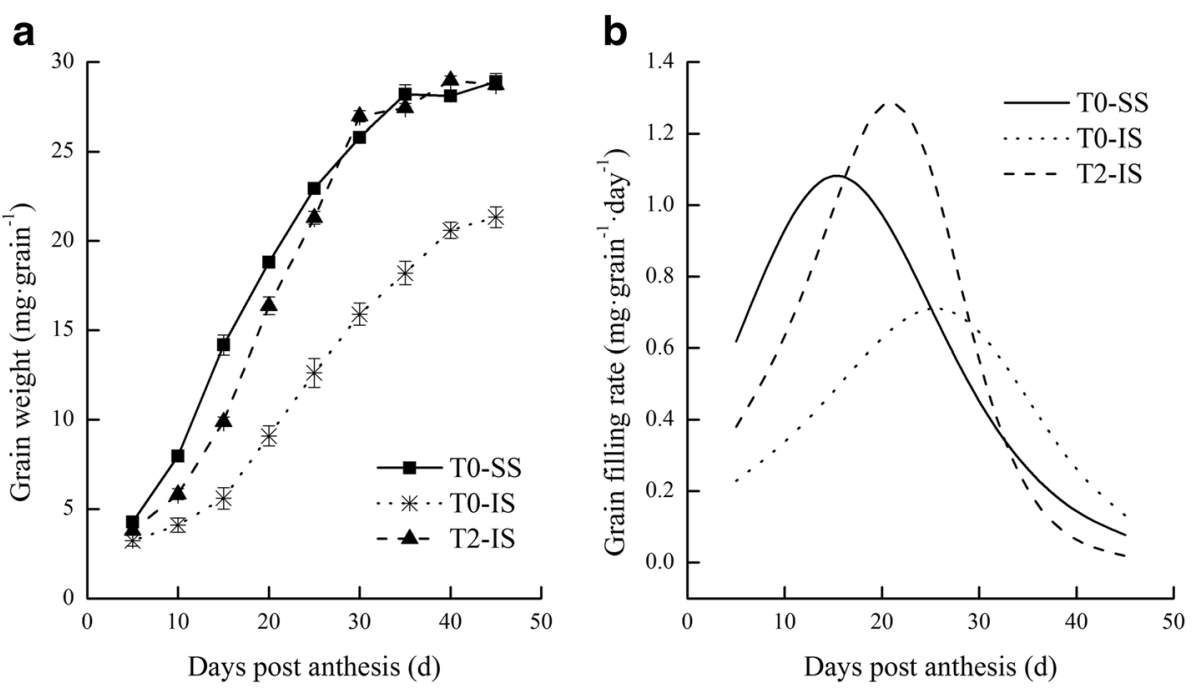

Fig. 1 Grain weight and grain filling rate of SS and IS of rice during grain-filling period. T0 represent control treatment with no spikelet thinning and $\mathrm{T} 2$ represent treatment with the upper 2/3 of spikelets were removed. The black square represent superior spikelets under the T0 treatment, the asterisk represent inferior spikelets under the T0 treatment, and the black triangle represent inferior spikelets under the T2 treatment. Vertical bars, where values exceed size of symbol, represent \pm SEM $(n=3)$

DEPs were identified, of which 63 proteins $(72.4 \%)$ were up-regulated and 24 proteins $(27.6 \%)$ were downregulated; and in the T2-IS/T0-IS comparison, 28 DEPs were identified, of which 22 proteins $(78.6 \%)$ were upregulated and 6 proteins $(21.4 \%)$ were down-regulated.

\section{Functional classification of DEPs between SS and IS under different treatments}

DEPs were classified according to their biological functions and were divided into 11 categories, including carbohydrate metabolism, protein metabolism, secondary metabolism, lipid metabolism, nucleotide metabolism, amino acid metabolism, photosynthesis, cell growth/division, material transport, signal transduction, and stress/defense (Table 2,
Fig. 4). In this study, proteins with unknown biological functions or those that could not be attributed to these 11 categories were classified into an unknown protein category. Among the 11 major functional categories, carbohydrate metabolism includes glucose metabolism, starch biosynthesis, glycolysis, tricarboxylic acid (TCA) cycle, and fermentation, while protein metabolism includes protein synthesis, proteolysis, protein folding, and storage.

The TO-SS/TO-IS comparison resulted in the greatest number of DEPs (159), which mainly participated in physiological and biochemical processes including carbohydrate metabolism (24.5\%), protein metabolism (20.13\%), stress/defense (8.18\%), and amino acid metabolism (9.43\%) (Fig. 4-a). Relatively fewer DEPs

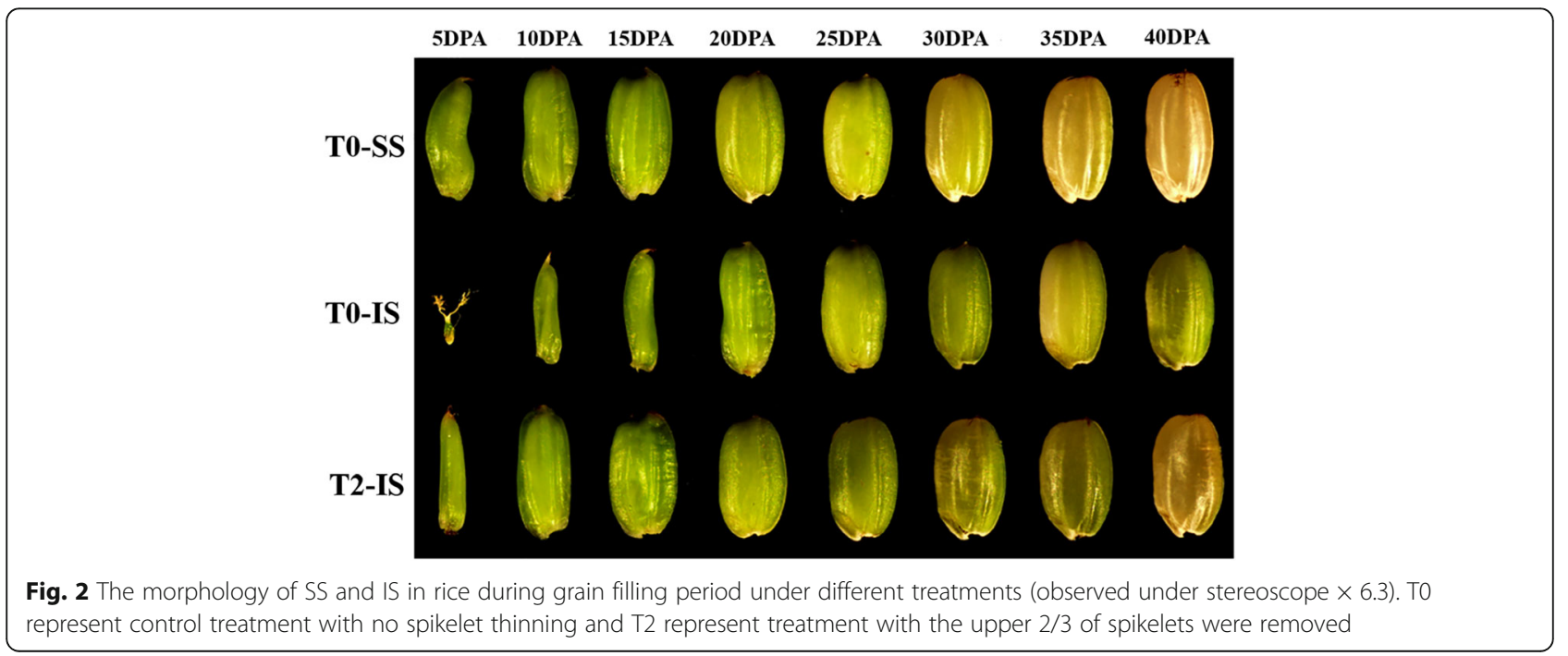


Table 2 Identification of 159, 87 and 28 differentially expressed proteins ( $\geq 1.5$ fold) between SS and IS at 10 DAP in group A (TO-SS/TO-IS), B (TO-SS/T2-IS) and C (T2-IS/TO-IS)

\begin{tabular}{|c|c|c|c|c|c|c|}
\hline \multirow[t]{2}{*}{ Protein No. } & \multirow[t]{2}{*}{ Accession } & \multirow{2}{*}{$\begin{array}{l}\text { Uniprot date } \\
\text { accession no. }\end{array}$} & \multirow{2}{*}{$\begin{array}{l}\text { Protein name } \\
\text { UniprotKB database }\end{array}$} & \multicolumn{3}{|c|}{ Fold-change ( $\geq 1.5$-fold) } \\
\hline & & & & TO-SS/TO-IS (A) & T0-SS/T2-IS (B) & T2-IS/T0-IS (C) \\
\hline \multicolumn{7}{|c|}{ Cell growth/division } \\
\hline 36 & Os02g0753800 & Q6Z6A7 & Annexin & $3.01 \pm 0.71$ & ns & ns \\
\hline 91 & Os05g0438800 & Q75HXO & Actin & $2.89 \pm 0.12$ & $1.76 \pm 0.08$ & ns \\
\hline 154 & Os07g0249700 & Q8H3C8 & IAA-amino acid hydrolase ILR1-like 8 & $3.52 \pm 0.47$ & $2.06 \pm 0.52$ & ns \\
\hline \multicolumn{7}{|c|}{ Sugar metabolism } \\
\hline 14 & Os03g0758100 & Q9AUV8 & Alpha-1,4 glucan phosphorylase & $4.70 \pm 0.48$ & $3.18 \pm 1.26$ & ns \\
\hline 19 & Os06g0194900 & P30298 & Sucrose synthase 2 & $6.04 \pm 1.05$ & ns & $3.57 \pm 1.79$ \\
\hline 26 & Os03g0278000 & Q8W3J0 & Os03g0278000 protein & $0.35 \pm 0.04$ & ns & ns \\
\hline 33 & Os03g0703000 & Q75193 & Beta-glucosidase 7 & $0.40 \pm 0.02$ & ns & ns \\
\hline 38 & Os01g0944700 & Q94CR1 & Beta 1,3-glucanase & $2.82 \pm 0.20$ & $2.45 \pm 0.27$ & ns \\
\hline 50 & Os10g0340600 & Q7XFK2 & Beta-galactosidase 14 & $0.40 \pm 0.13$ & ns & ns \\
\hline 54 & Os02g0752200 & Q6Z8I7 & Os02g0752200 protein & $0.37 \pm 0.09$ & ns & ns \\
\hline 65 & Os08g0509200 & Q84YK7 & Beta-glucosidase 27 & $0.45 \pm 0.02$ & ns & $0.57 \pm 0.02$ \\
\hline 121 & Os06g0172800 & Q5SNC5 & Putative seed imbibition protein & $2.52 \pm 0.32$ & ns & ns \\
\hline 136 & Os03g0340500 & Q10LP5 & Sucrose synthase 4 & $5.38 \pm 1.07$ & $2.02 \pm 0.19$ & ns \\
\hline \multicolumn{7}{|c|}{ Starch biosynthesis } \\
\hline 6 & Os04g0409200 & Q0JDF0 & Os04g0409200 protein & $0.48 \pm 0.04$ & ns & ns \\
\hline 40 & Os08g0345800 & P15280 & $\begin{array}{l}\text { Glucose-1-phosphate adenylyltransferase } \\
\text { small subunit, chloroplastic/amyloplastic }\end{array}$ & $4.66 \pm 0.51$ & ns & ns \\
\hline 41 & Os05g0580000 & Q688T8 & Glucose-1-phosphate adenylyltransferase & $0.21 \pm 0.08$ & $0.21 \pm 0.08$ & ns \\
\hline 42 & Os01g0130400 & Q9LGC6 & Putative alpha-glucosidase & $0.48 \pm 0.04$ & ns & ns \\
\hline 44 & Os01g0633100 & Q7G065 & ADP-glucose pyrophosphorylase/AGPase & $3.36 \pm 0.44$ & ns & ns \\
\hline 45 & Os01g0894300 & Q0JGZ6 & Fructokinase-1 & $0.53 \pm 0.04$ & ns & ns \\
\hline 46 & Os01g0841600 & Q8LR75 & Triosephosphate isomerase & ns & $0.33 \pm 0.05$ & ns \\
\hline 49 & Os06g0675700 & Q0DA62 & Probable alpha-glucosidase Os06g0675700 & $4.71 \pm 1.86$ & $2.51 \pm 0.76$ & ns \\
\hline 84 & Os09g0553200 & Q93X08 & Os09g0553200 protein & $3.23 \pm 0.63$ & ns & ns \\
\hline 86 & Os05g0482700 & Q5KQH5 & $\begin{array}{l}\text { Putative 2,3-bisphosphoglycerate-independent } \\
\text { phosphoglycerate mutase }\end{array}$ & $0.60 \pm 0.05$ & ns & ns \\
\hline 94 & Os08g0520900 & Q0J4C6 & Os08g0520900 protein & $3.19 \pm 0.38$ & $2.50 \pm 0.36$ & ns \\
\hline 118 & Os08g0191433 & Q6Z1D6 & Putative starch synthase DULL1 & $2.45 \pm 0.16$ & ns & $2.43 \pm 0.19$ \\
\hline 132 & Os04g0164900 & Q7X834 & OSJNBa0019G23.2 protein/pullulanase & $20.08 \pm 1.58$ & $5.66 \pm 0.45$ & $3.84 \pm 0.56$ \\
\hline 152 & Os04g0526600 & Q0JBLO & Alpha-amylase/subtilisin inhibitor & $6.59 \pm 0.45$ & $3.02 \pm 0.06$ & ns \\
\hline 158 & Os02g0528200 & Q6H6P8 & Branching enzyme-3/SBE3 & $2.36 \pm 0.36$ & ns & ns \\
\hline 159 & Os06g0726400 & Q0D9D0 & Os06g0726400 protein/SBE1 & $15.54 \pm 0.72$ & $3.34 \pm 0.24$ & $5.00 \pm 0.42$ \\
\hline \multicolumn{7}{|c|}{ Respration (Glycolysis,TCA and Fermentation) } \\
\hline 4 & Os01g0905800 & Q5N725 & Fructose-bisphosphate aldolase & $3.99 \pm 0.62$ & ns & ns \\
\hline 27 & Os01g0926300 & Q5JK10 & Os01g0926300 protein & $0.37 \pm 0.07$ & ns & ns \\
\hline 30 & Os02g0601300 & Q6K5G8 & $\begin{array}{l}\text { Glyceraldehyde-3-phosphate } \\
\text { dehydrogenase 3, cytosolic }\end{array}$ & $3.76 \pm 0.30$ & ns & ns \\
\hline 59 & Os02g0169300 & Q6H6C7 & Phosphoglycerate kinase & $4.84 \pm 0.51$ & ns & ns \\
\hline 69 & Os06g0668200 & Q655T1 & Phosphoglycerate kinase & $2.56 \pm 0.42$ & ns & ns \\
\hline 76 & Os10g0478200 & Q7XDC8 & Malate dehydrogenase, cytoplasmic & $3.51 \pm 0.12$ & ns & ns \\
\hline 82 & Os08g0191700 & Q0J7H9 & Lactoylglutathione lyase & $3.30 \pm 0.40$ & $3.14 \pm 1.07$ & ns \\
\hline
\end{tabular}


Table 2 Identification of 159, 87 and 28 differentially expressed proteins ( $\geq 1.5$ fold) between SS and IS at 10 DAP in group A (TO-SS/TO-IS), B (TO-SS/T2-IS) and C (T2-IS/TO-IS) (Continued)

\begin{tabular}{|c|c|c|c|c|c|c|}
\hline 89 & Os11g0210500 & Q0ITW7 & Alcohol dehydrogenase 2 & $5.38 \pm 1.61$ & ns & ns \\
\hline 104 & Os06g0486800 & Q0DC43 & Formate dehydrogenase & $3.25 \pm 0.54$ & $2.41 \pm 0.74$ & ns \\
\hline 120 & Os08g0545200 & Q6ZBH2 & $\begin{array}{l}\text { Os08g0545200 protein/ } \\
\text { Sorbitol dehydrogenase }\end{array}$ & $12.37 \pm 2.25$ & $2.49 \pm 0.24$ & $5.09 \pm 0.97$ \\
\hline 122 & Os03g0293500 & Q10MW3 & Pyruvate decarboxylase 2 & $3.96 \pm 0.28$ & $2.72 \pm 1.11$ & ns \\
\hline 125 & Os06g0326400 & Q69T78 & $\begin{array}{l}\text { Pyrophosphate-fructose 6-phosphate } \\
\text { 1-phosphotransferase subunit alpha }\end{array}$ & $2.05 \pm 0.21$ & ns & $2.27 \pm 0.28$ \\
\hline 127 & Os07g0187200 & Q7XI14 & $\begin{array}{l}\text { Probable D-2-hydroxyglutarate } \\
\text { dehydrogenase, mitochondrial }\end{array}$ & $4.60 \pm 0.81$ & ns & ns \\
\hline 173 & Os04g0486950 & Q7XUG1 & Malate synthase & $5.85 \pm 1.70$ & $4.64 \pm 0.51$ & ns \\
\hline \multicolumn{7}{|c|}{ Photosynthesis } \\
\hline 1 & Os01g0711000 & Q8S7T5 & ATP synthase subunit alpha & ns & $4.23 \pm 1.46$ & ns \\
\hline 3 & Os10g0356000 & P0C512 & Ribulose bisphosphate carboxylase large chain & $2.74 \pm 0.09$ & $2.40 \pm 0.75$ & ns \\
\hline 7 & Q8S6G5[a] & Q8S6G5 & Photosystem II CP43 reaction center protein & ns & $1.92 \pm 0.09$ & ns \\
\hline 23 & Q69VC8[b] & Q69VC8 & Photosystem II CP47 reaction center protein & ns & $3.17 \pm 1.27$ & ns \\
\hline 43 & Os03g0563300 & Q53RM0 & Magnesium-chelatase subunit Chll, chloroplastic & $0.46 \pm 0.01$ & ns & ns \\
\hline 57 & Os10g0492000 & Q9FWV2 & Putative chloroplast inner envelope protein & $2.36 \pm 0.57$ & ns & ns \\
\hline \multicolumn{7}{|c|}{ Material transport } \\
\hline 22 & Os07g0448800 & Q8H5N9 & Probable aquaporin PIP2-1 & ns & $3.21 \pm 0.71$ & ns \\
\hline 64 & Os08g0513600 & Q6Z8M9 & Os08g0513600 protein & $6.26 \pm 2.17$ & ns & ns \\
\hline 75 & Os02g0202400 & Q6Z782 & Os02g0202400 protein & $3.04 \pm 0.84$ & ns & ns \\
\hline 78 & Os11g0644100 & Q2R016 & Leucine Rich Repeat family protein, expressed & $0.24 \pm 0.02$ & ns & ns \\
\hline 111 & Os03g0271200 & Q10NF2 & Protein TOC75, chloroplastic & $4.63 \pm 0.36$ & $1.95 \pm 0.32$ & $2.45 \pm 0.41$ \\
\hline 123 & Os05g0111200 & Q65XV6 & Os05g0111200 protein & $3.77 \pm 0.18$ & $2.85 \pm 0.23$ & ns \\
\hline 126 & Os03g0240500 & Q10PB3 & Translocase of chloroplast & $2.27 \pm 0.17$ & ns & ns \\
\hline \multicolumn{7}{|c|}{ Signal transduction } \\
\hline 2 & Os03g0710800 & Q10E23 & 14-3-3-like protein GF14-F & $0.53 \pm 0.01$ & ns & ns \\
\hline 101 & Os06g0110100 & Q8H684 & OSEYA1 & $4.56 \pm 1.10$ & $1.86 \pm 0.18$ & $2.51 \pm 0.58$ \\
\hline 129 & Os01g0356800 & Q0JMV9 & Os01g0356800 protein/ GTP binding protein & $35.53 \pm 10.52$ & $2.45 \pm 0.06$ & $12.89 \pm 1.52$ \\
\hline 138 & Os02g0799000 & Q69QZ0 & Probable protein phosphatase $2 C 27$ & $5.05 \pm 0.74$ & ns & ns \\
\hline \multicolumn{7}{|c|}{ Stress and defense } \\
\hline 5 & Os07g0186000 & Q0D840 & Thioredoxin $\mathrm{H} 1$ & ns & $3.65 \pm 0.82$ & ns \\
\hline 8 & Os02g0115700 & Q0E4K1 & Catalase isozyme A & $0.28 \pm 0.01$ & ns & $0.49 \pm 0.07$ \\
\hline 10 & Os05g0116100 & Q65XA0 & Dehydroascorbate reductase & $2.15 \pm 0.13$ & $2.97 \pm 0.40$ & ns \\
\hline 11 & Os05g0323900 & Q43008 & Superoxide dismutase $[\mathrm{Mn}]$, mitochondrial & $1.77 \pm 0.05$ & $3.29 \pm 0.33$ & ns \\
\hline 21 & Os04g0508300 & P55142 & Glutaredoxin-C6 & $2.95 \pm 0.11$ & $3.44 \pm 0.66$ & ns \\
\hline 24 & Os05g0157200 & Q75M01 & Os05g0157200 protein & ns & $2.97 \pm 0.28$ & ns \\
\hline 35 & Os12g0244100 & Q2QV45 & $70 \mathrm{kDa}$ heat shock protein & $5.12 \pm 1.21$ & $2.18 \pm 0.62$ & ns \\
\hline 58 & Os05g0453700 & Q7XXS5 & Os05g0453700 protein & $4.37 \pm 0.28$ & $3.23 \pm 0.16$ & ns \\
\hline 67 & Os07g0624600 & Q7XI41 & Probable trehalose-phosphate phosphatase 3 & $0.35 \pm 0.03$ & $0.29 \pm 0.08$ & ns \\
\hline 73 & Os12g0514500 & Q0IN14 & Hsp90 protein, expressed & $0.36 \pm 0.05$ & $0.32 \pm 0.05$ & ns \\
\hline 81 & Os01g0270100 & Q0JNR2 & Cysteine proteinase inhibitor 12 & $2.14 \pm 0.21$ & ns & ns \\
\hline 113 & Os07g0694700 & Q0D3B8 & Ascorbate peroxidase & $0.51 \pm 0.06$ & ns & ns \\
\hline
\end{tabular}


Table 2 Identification of 159, 87 and 28 differentially expressed proteins ( $\geq 1.5$ fold) between SS and IS at 10 DAP in group A (TO-SS/TO-IS), B (T0-SS/T2-IS) and C (T2-IS/TO-IS) (Continued)

\begin{tabular}{|c|c|c|c|c|c|c|}
\hline 134 & Os01g0663400 & Q0JKM8 & Os01g0663400 protein & $8.82 \pm 0.50$ & $2.32 \pm 0.43$ & $3.68 \pm 0.78$ \\
\hline 166 & Os07g0638300 & P0C5C9 & 1-Cys peroxiredoxin $\mathrm{A}$ & $14.91 \pm 5.52$ & $3.85 \pm 0.39$ & $3.59 \pm 1.15$ \\
\hline 174 & Os03g0245800 & Q10P60 & 26.7 kDa heat shock protein, chloroplastic & $4.84 \pm 0.88$ & $2.16 \pm 0.53$ & ns \\
\hline \multicolumn{7}{|c|}{ Protein synthesis and destination } \\
\hline 12 & Os04g0685200 & Q7XPU2 & OSJNBa0088H09.14 protein & $0.38 \pm 0.04$ & ns & ns \\
\hline 16 & Os06g0687700 & Q653F6 & Putative t-complex protein 1 theta chain & $0.40 \pm 0.01$ & ns & ns \\
\hline 25 & Os12g0277500 & Q2QU06 & 60 kDa chaperonin alpha subunit & $0.21 \pm 0.03$ & $0.23 \pm 0.05$ & ns \\
\hline 34 & Os03g0804800 & Q75HJ3 & $\begin{array}{l}\text { Putative TCP-1/cpn60 chaperonin } \\
\text { family protein }\end{array}$ & $0.50 \pm 0.06$ & $0.61 \pm 0.10$ & ns \\
\hline 48 & Os02g0332200 & Q6YUK5 & T-complex protein 1 subunit delta & $0.27 \pm 0.03$ & $0.32 \pm 0.05$ & ns \\
\hline 68 & Os07g0578300 & Q6ZL89 & Os07g0578300 protein & $0.05 \pm 0.01$ & $0.09 \pm 0.03$ & $0.38 \pm 0.01$ \\
\hline 71 & Os03g0619400 & Q6AV23 & $\begin{array}{l}\text { Putative TCP-1/cpn60 chaperonin } \\
\text { family protein }\end{array}$ & $0.40 \pm 0.05$ & ns & ns \\
\hline 79 & Os02g0717400 & Q6ZGV8 & Clustered mitochondria protein homolog & ns & $0.30 \pm 0.08$ & ns \\
\hline 95 & Os03g0565500 & Q10139 & $\begin{array}{l}\text { Elongation factor } \mathrm{G} \text {, mitochondrial, } \\
\text { putative, expressed }\end{array}$ & $0.32 \pm 0.08$ & $0.33 \pm 0.08$ & ns \\
\hline 103 & Os09g0491772 & B9G4B3 & Os09g0491772 protein & $0.16 \pm 0.04$ & $0.15 \pm 0.04$ & ns \\
\hline 110 & Os02g0100100 & Q67IX6 & Protein disulfide isomerase-like $1-4$ & $5.62 \pm 0.64$ & ns & $3.37 \pm 0.67$ \\
\hline 117 & Os01g0185200 & Q5VRX8 & Os01g0185200 protein & $3.21 \pm 0.26$ & ns & ns \\
\hline 119 & Os01g0752700 & Q5JMX4 & Os01g0752700 protein & $0.31 \pm 0.04$ & $0.28 \pm 0.02$ & ns \\
\hline 130 & Os09g0451500 & Q67UF5 & Protein disulfide isomerase-like $2-3$ & $2.15 \pm 0.47$ & ns & ns \\
\hline 135 & Os02g0506500 & Q6K6K7 & Ubiquitin-like modifier-activating enzyme 5 & $3.28 \pm 1.07$ & ns & ns \\
\hline 143 & Os09g0252800 & Q6K3Y7 & Putative ubiquitin-protein ligase 1 & ns & $0.53 \pm 0.04$ & ns \\
\hline 144 & Os02g0115900 & Q6Z7B0 & Dnak-type molecular chaperone Bip & $6.30 \pm 1.31$ & ns & $3.84 \pm 1.04$ \\
\hline 147 & Os05g0557200 & Q61605 & Os05g0557200 protein & $2.13 \pm 0.15$ & ns & ns \\
\hline 148 & Os07g0215500 & Q0D7S0 & Allergen RA5B & $22.26 \pm 5.08$ & $4.59 \pm 0.37$ & ns \\
\hline 153 & Os07g0213800 & Q8H4M4 & Allergenic protein & ns & $5.68 \pm 0.20$ & ns \\
\hline 155 & Os11g0199200 & Q53LQ0 & Protein disulfide isomerase-like 1-1 & $4.62 \pm 0.40$ & ns & ns \\
\hline 161 & Os03g0610650 & Q75H81 & Serpin-ZXA & $3.53 \pm 0.52$ & $2.30 \pm 0.33$ & ns \\
\hline 170 & Os05g0519700 & Q6F2Y7 & Chaperone protein ClpB1 & $4.54 \pm 0.18$ & $2.35 \pm 0.39$ & $1.98 \pm 0.35$ \\
\hline \multicolumn{7}{|c|}{ Storage proteins } \\
\hline 97 & Os07g0609000 & Q6YTX6 & Seed protein & $19.17 \pm 2.41$ & $6.65 \pm 2.22$ & ns \\
\hline 99 & Os01g0762500 & Q0JJ36 & Glutelin & $1.54 \pm 0.02$ & ns & ns \\
\hline 128 & Os03g0793700 & Q852L2 & Cupin family protein, expressed & $8.40 \pm 1.03$ & $4.63 \pm 0.42$ & ns \\
\hline 141 & Os05g0499100 & Q0DH05 & Alpha-globulin & $39.03 \pm 4.70$ & $4.02 \pm 0.47$ & ns \\
\hline 145 & Os02g0242600 & Q6ESW6 & Glutelin & $19.54 \pm 5.20$ & ns & $9.51 \pm 1.33$ \\
\hline 151 & Os03g0197300 & QODUA3 & Os03g0197300 protein & $25.94 \pm 10.52$ & $6.59 \pm 1.98$ & ns \\
\hline 157 & Os02g0456100 & Q6K7K6 & Glutelin & $36.64 \pm 4.06$ & $3.66 \pm 0.31$ & $11.87 \pm 0.71$ \\
\hline 163 & Os03g0336100 & Q0DS36 & Os03g0336100 protein & $23.65 \pm 1.52$ & $3.60 \pm 0.61$ & ns \\
\hline 165 & Os02g0249900 & Q0E2D2 & Glutelin & ns & $4.24 \pm 0.89$ & ns \\
\hline 167 & Os07g0214300 & Q0D7S4 & Seed allergenic protein RAG2 & $12.44 \pm 2.51$ & $6.26 \pm 0.42$ & ns \\
\hline 169 & Os02g0249000 & Q6K508 & Glutelin & $28.90 \pm 5.78$ & $1.86 \pm 0.30$ & $14.62 \pm 1.20$ \\
\hline 171 & Os03g0663800 & Q75GX9 & Cupin family protein, expressed & $31.11 \pm 3.49$ & ns & ns \\
\hline 172 & Os02g0268300 & Q0E261 & Glutelin & $56.76 \pm 1.25$ & $4.28 \pm 0.71$ & ns \\
\hline
\end{tabular}


Table 2 Identification of 159, 87 and 28 differentially expressed proteins ( $\geq 1.5$ fold) between SS and IS at 10 DAP in group A (TO-SS/TO-IS), B (TO-SS/T2-IS) and C (T2-IS/TO-IS) (Continued)

\begin{tabular}{|c|c|c|c|c|c|c|}
\hline \multicolumn{7}{|c|}{ Amino acid metabolism } \\
\hline 9 & Os08g0447000 & Q6ZAA5 & D-3-phosphoglycerate dehydrogenase & $0.18 \pm 0.06$ & $0.28 \pm 0.11$ & ns \\
\hline 20 & Os11g0216900 & Q0ITU1 & Methylthioribose-1-phosphate isomerase & $0.31 \pm 0.01$ & ns & ns \\
\hline 52 & Os03g0738400 & Q7Y1F0 & Serine hydroxymethyltransferase & $1.99 \pm 0.05$ & ns & ns \\
\hline 66 & Os03g0223400 & Q10PS4 & Glutamine synthetase & $5.20 \pm 0.10$ & $3.93 \pm 1.24$ & ns \\
\hline 70 & Os09g0255400 & Q8H3R5 & $\begin{array}{l}\text { Putative indole-3-glycerol } \\
\text { phosphate synthase }\end{array}$ & $3.82 \pm 0.69$ & ns & ns \\
\hline 85 & Os12g0235800 & Q2QVC1 & $\begin{array}{l}\text { Argininosuccinate synthase, } \\
\text { chloroplast, putative, expressed }\end{array}$ & $2.74 \pm 0.13$ & ns & ns \\
\hline 92 & Os03g0136200 & Q10S41 & Methyltransferase & $0.42 \pm 0.04$ & ns & ns \\
\hline 93 & Os12g0607000 & Q2QME6 & Homocysteine S-methyltransferase 3 & $3.83 \pm 0.20$ & ns & ns \\
\hline 105 & Os12g0138900 & Q2QXY9 & $\begin{array}{l}\text { 2-isopropylmalate synthase B, } \\
\text { putative, expressed }\end{array}$ & $2.75 \pm 0.17$ & $1.81 \pm 0.27$ & ns \\
\hline 114 & Os02g0783625 & Q6K7D6 & $\begin{array}{l}\text { Putative lysine-ketoglutarate reductase/ } \\
\text { saccharopine dehydrogenase bifunctional enzyme }\end{array}$ & $3.13 \pm 0.56$ & ns & ns \\
\hline 140 & Os12g0145100 & Q2QXS4 & Os12g0145100 protein & ns & ns & $2.63 \pm 0.68$ \\
\hline 142 & Os10g0390500 & Q94HC5 & Putative alanine amino transferase & $3.07 \pm 0.38$ & ns & $3.02 \pm 0.44$ \\
\hline 149 & Os12g0578200 & Q2QN58 & $\begin{array}{l}\text { Chorismate mutase, chloroplast, } \\
\text { putative, expressed }\end{array}$ & $18.67 \pm 2.19$ & $1.92 \pm 0.11$ & $8.26 \pm 0.90$ \\
\hline 150 & Os03g0171900 & Q10R45 & $\begin{array}{l}\text { Alanine-glyoxylate aminotransferase } 2 \text {, } \\
\text { mitochondrial, putative, expressed }\end{array}$ & $3.68 \pm 0.78$ & ns & ns \\
\hline 160 & Os04g0389800 & Q0E0Z3 & Acetolactate synthase & $3.41 \pm 0.30$ & $1.80 \pm 0.24$ & ns \\
\hline 162 & Os01g0760600 & Q0JJ47 & Aspartate aminotransferase & $3.82 \pm 0.36$ & ns & $3.30 \pm 0.31$ \\
\hline \multicolumn{7}{|c|}{ Nucleotides } \\
\hline 29 & Os10g0539500 & Q7XUC9 & Histone $\mathrm{H} 4$ & $0.39 \pm 0.03$ & ns & ns \\
\hline 37 & Os01g0550000 & Q5JK84 & DEAD-box ATP-dependent RNA helicase 15 & $0.54 \pm 0.06$ & ns & ns \\
\hline 60 & Os01g0275600 & Q9SDG8 & Protein argonaute $4 \mathrm{~A}$ & $0.32 \pm 0.06$ & $0.43 \pm 0.09$ & ns \\
\hline 63 & Os02g0736400 & Q6Z744 & Dihydropyrimidine dehydrogenase & $2.25 \pm 0.17$ & ns & ns \\
\hline 87 & Os03g0158500 & Q8H8C1 & Putative RNA-binding protein & ns & $0.31 \pm 0.05$ & ns \\
\hline 88 & Os02g0214500 & Q6H8A9 & NAC6 & $4.80 \pm 0.64$ & ns & ns \\
\hline 102 & Os07g0471300 & Q69UP6 & Protein argonaute 18 & $0.37 \pm 0.08$ & $0.41 \pm 0.09$ & ns \\
\hline 107 & Os02g0137400 & Q6YXY3 & Putative splicing factor $3 \mathrm{~b}$, subunit 3, $130 \mathrm{kDa}$ & $0.56 \pm 0.03$ & ns & ns \\
\hline 109 & Os02g0821800 & Q6AT27 & Putative fibrillarin & $0.42 \pm 0.08$ & ns & ns \\
\hline 115 & Os07g0212300 & Q8H4U7 & Mut T-like protein & $10.59 \pm 1.42$ & ns & ns \\
\hline 131 & Os02g0523500 & Q6H547 & Os02g0523500 protein & $2.18 \pm 0.24$ & ns & $1.95 \pm 0.34$ \\
\hline \multicolumn{7}{|c|}{ Lipid metabolism } \\
\hline 28 & Os05g0295300 & B9FK36 & Acetyl-CoA carboxylase 2 & $0.64 \pm 0.01$ & ns & ns \\
\hline 51 & Os11g0558300 & Q2R2L5 & $\begin{array}{l}\text { AMP-binding enzyme family } \\
\text { protein, expressed }\end{array}$ & $0.34 \pm 0.14$ & ns & ns \\
\hline 56 & Os03g0181500 & Q8H7L2 & 3-ketoacyl-CoA synthase & $0.17 \pm 0.02$ & $0.25 \pm 0.01$ & ns \\
\hline 62 & Os06g0156700 & Q5VMA4 & Os06g0156700 protein & $3.49 \pm 0.43$ & ns & ns \\
\hline 77 & Os05g0567100 & Q0DFW1 & Aspartic proteinase oryzasin 1 & $2.58 \pm 0.49$ & $2.54 \pm 0.40$ & ns \\
\hline 96 & Os01g0880800 & Q8LJJ9 & $\begin{array}{l}\text { Stearoyl-[acyl-carrier-protein] } \\
\text { 9-desaturase 1, chloroplastic }\end{array}$ & ns & $0.35 \pm 0.05$ & ns \\
\hline 108 & Os07g0188800 & Q6Z4E4 & $\begin{array}{l}\text { Methylmalonate semi-aldehyde } \\
\text { dehydrogenase }\end{array}$ & $2.13 \pm 0.19$ & $2.04 \pm 0.40$ & ns \\
\hline
\end{tabular}


Table 2 Identification of 159, 87 and 28 differentially expressed proteins ( $\geq 1.5$ fold) between SS and IS at 10 DAP in group A (TO-SS/TO-IS), B (TO-SS/T2-IS) and C (T2-IS/TO-IS) (Continued)

\begin{tabular}{|c|c|c|c|c|c|c|}
\hline 124 & Os06g0260500 & Q5Z7E7 & 3-ketoacyl-CoA synthase & $0.24 \pm 0.10$ & $0.26 \pm 0.12$ & ns \\
\hline 146 & Os01g0348600 & Q94CN1 & Os01g0348600 protein & $0.38 \pm 0.15$ & ns & ns \\
\hline \multicolumn{7}{|c|}{ Secondary metabolism } \\
\hline 31 & Os07g0529600 & Q7XXS4 & Thiamine biosynthetic enzyme & $0.30 \pm 0.02$ & $0.30 \pm 0.02$ & ns \\
\hline 47 & Os08g0157500 & Q6ZD89 & Flavone 3'-O-methyltransferase 1 & $4.73 \pm 0.80$ & $2.59 \pm 0.69$ & ns \\
\hline 55 & Os08g0498400 & Q7F8T6 & Tricin synthase 2 & $3.56 \pm 0.56$ & $2.19 \pm 0.30$ & ns \\
\hline 116 & Os09g0446800 & Q0J1E1 & Os09g0446800 protein & $2.55 \pm 0.03$ & $1.67 \pm 0.18$ & ns \\
\hline 139 & Os03g0192700 & Q10QK8 & Inositol-3-phosphate synthase & $2.06 \pm 0.18$ & ns & ns \\
\hline 164 & Os08g0189100 & Q6YZA9 & Germin-like protein 8-2 & $7.61 \pm 0.71$ & $5.34 \pm 0.86$ & ns \\
\hline \multicolumn{7}{|c|}{ Unknown } \\
\hline 13 & Os12g0555500 & Q2QNS7 & Os12g0555500 protein & $0.49 \pm 0.03$ & ns & ns \\
\hline 15 & Os06g0646500 & Q67W57 & Os06g0646500 protein & ns & ns & $0.57 \pm 0.04$ \\
\hline 17 & Os03g0278200 & Q10N92 & Os03g0278200 protein & $0.46 \pm 0.05$ & ns & ns \\
\hline 18 & Os11g0687100 & Q2QZH3 & Os11g0687100 protein & $0.08 \pm 0.03$ & $0.21 \pm 0.11$ & $0.31 \pm 0.05$ \\
\hline 32 & Os12g0182200 & Q2QWU7 & $\begin{array}{l}\text { Dihydrolipoamide S-acetyltransferase, } \\
\text { putative, expressed }\end{array}$ & $0.53 \pm 0.05$ & ns & ns \\
\hline 39 & Os06g0613000 & Q69WY2 & Uncharacterized protein & $0.36 \pm 0.04$ & ns & ns \\
\hline 53 & Os01g0916600 & Q7F2X8 & Os01g0916600 protein/OsGRP2 & $0.24 \pm 0.01$ & $0.28 \pm 0.01$ & ns \\
\hline 61 & Os11g0687200 & Q2QZH2 & Expressed protein & $0.30 \pm 0.05$ & ns & $0.54 \pm 0.03$ \\
\hline 72 & Os07g0409100 & Q7XTM4 & OSJNBa0033G05.21 protein & $0.53 \pm 0.05$ & ns & ns \\
\hline 74 & Os07g0638100 & Q8GVH2 & Os07g0638100 protein & $0.53 \pm 0.03$ & ns & ns \\
\hline 80 & Os01g0128400 & Q9LGA3 & Os01g0128400 protein & $2.00 \pm 0.06$ & ns & ns \\
\hline 83 & Os04g0531900 & Q7X8W6 & OSJNBa0081C01.20 protein & $5.73 \pm 1.54$ & $4.34 \pm 0.33$ & ns \\
\hline 90 & Os10g0463200 & Q8H906 & $\begin{array}{l}\text { Putative early nodulin gene } \\
\text { (Enod) related protein }\end{array}$ & $0.36 \pm 0.03$ & $0.46 \pm 0.04$ & ns \\
\hline 98 & Os03g0327600 & Q10M12 & Expressed protein & $2.03 \pm 0.08$ & ns & ns \\
\hline 100 & Os07g0568700 & Q0D5C7 & Os07g0568700 protein & $0.44 \pm 0.03$ & ns & ns \\
\hline 106 & Os04g0482800 & Q7XUP3 & OSJNBb0011N17.20 protein & $3.73 \pm 0.15$ & $2.14 \pm 0.10$ & ns \\
\hline 112 & Os02g0783700 & Q0DX00 & Os02g0783700 protein & $2.05 \pm 0.19$ & ns & ns \\
\hline 133 & Os05g0132100 & Q0DL03 & Os05g0132100 protein & $0.34 \pm 0.05$ & ns & ns \\
\hline 137 & Os06g0214300 & Q69Y21 & Os06g0214300 protein & $2.85 \pm 0.43$ & $1.68 \pm 0.16$ & ns \\
\hline 156 & Os04g0404400 & Q7X618 & OJ000315_02.8 protein & $16.73 \pm 7.88$ & $5.11 \pm 1.18$ & ns \\
\hline 168 & Os10g0463800 & Q337M4 & Os10g0463800 protein & $3.60 \pm 0.15$ & $2.59 \pm 0.29$ & ns \\
\hline
\end{tabular}

Accession: the code of the identified protein in RAP database (http://rapdb.dna.affrc.go.jp/); [a] and [b]: the code of these two identified proteins in RAP database were not found; ns means no significant change of protein abundance between the two compared samples; T0: control treatment with no spikelet thinning; T2: the upper 2/3 of spikelets were removed. SS: Superior spikelets; IS: Inferior spikelets; Values are means \pm S.D. of two replications. The screening criteria for differentially expressed proteins was a fold change $>1.5$ or $<0.67$ and a $p$-value $<0.05$

were identified in the T0-SS/T2-IS comparison (87), and these mainly participated in the same metabolic processes as those in the T0-SS/T0-IS comparison. Among these, $17.24 \%$ were related to carbohydrate metabolism and $26.44 \%$ were associated with protein metabolism (Fig. 4-b). The T2-IS/T0-IS comparison resulted in the fewest DEPs (28), but $25 \%$ of these were involved in carbohydrate metabolism and 25\% were involved in protein metabolism. Moreover, 7.14\% of DEPs in this group were associated with signal transduction, which was significantly more than $2.52 \%$ in the T0-SS/T0-IS comparison and $2.3 \%$ in the T0SS/T2-IS comparison (Fig. 4-c).

Together, the above experimental results showed that carbohydrate metabolism and protein metabolism play key roles in the differential development of SS and IS. Among the three comparisons, starch synthesis and protein storage functions were relatively prevalent among carbohydrate metabolism and protein metabolism functions (Fig. 4), suggesting that the supply of 


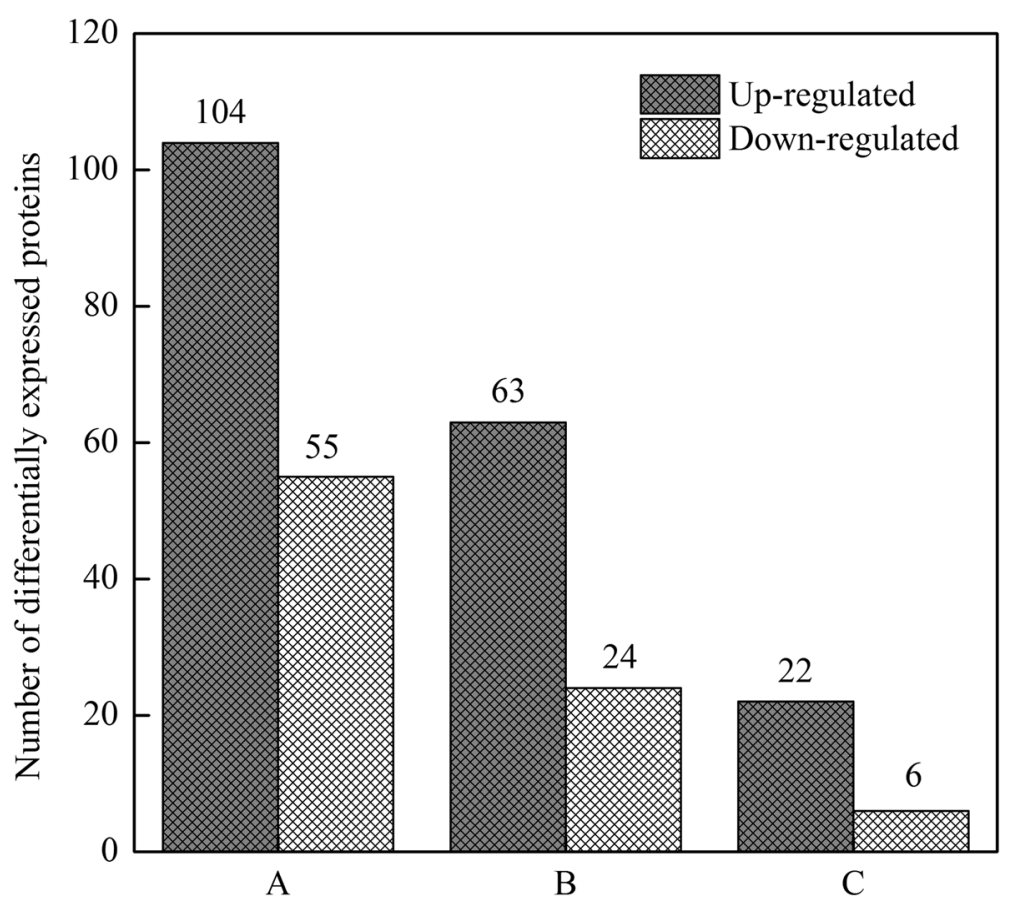

Fig. 3 Patterns of change on differentially expressed proteins of a (TO-SS / TO-IS), b (TO-SS / T2-IS) and $\mathbf{c}$ (T2-IS / TO-IS). T0 represent control treatment with no spikelet thinning and $\mathrm{T} 2$ represent treatment with the upper $2 / 3$ of spikelets were removed

carbohydrates to IS increased after SS removing [16], starch and protein synthesis in the grains are significantly enhanced. It is worth noting that the signal transduction function showed the greatest influence on IS development after SS removal, which may be one of the reasons for the increase in IS grain filling after SS removal treatment.

\section{Discussion}

\section{Physiological differences between SS and IS under different treatments}

The phenomena of low seed setting rates and poor plumpness are common in large-panicle rice varieties, and this is mainly due to poor IS grain filling and the formation of empty and blighted grains of rice [31]. These phenomena were also observed in this study. The grain weight and seed setting rate were significant different between SS and IS. SS elongated rapidly and grew well at $10 \mathrm{DPA}$, while IS were in a state of developmental stagnation. After the removal of SS, the IS grain size and grain weight significantly increased, indicating that 10 DPA was the end of the stagnant grain filling period and the beginning of the grain filling initiation period. Limited assimilate supply was generally considered to be the main cause of poor IS grain filling [7, 11]. The results from our previous studies [16] and this study support this view as well, as SS removal significantly improved IS grain size, sucrose content, grain weight, and grain filling rate in W1844. Since grain filling is a highly complex process, its molecular mechanisms need to be further elucidated.

Low expression proteins associated with endosperm cell growth and division leading to small sink capacity

A positive correlation between endosperm cell numbers and grain weight has been found in rice [32], wheat [33], and maize [34]. Previous reports showed that SS had a large number of endosperm cells, and thus a large sink size [5]. However, IS endosperm cell division was stagnant at the early grain filling stage, which limited IS sink establishment. In the present study, three cell divisionrelated proteins, actin, annexin, and IAA-amino acid hydrolase ILR1-like 8 were identified. Significant differences in the expressions of these three proteins between SS and IS were considered to be very important for endosperm cell division.

The actin cytoskeleton provides a structural framework for defining cell shape and polarity. Its dynamic properties provide the driving force for cells to move and to divide [35]. Annexins are thought to be associated with cell proliferation and differentiation [36]. In this study, actin and annexin were identifed and their abundances in T0-SS showed significantly higher up-regulation compared with those in T0-IS, this matched well with differences in endosperm cell division between SS and IS. Although SS removal treatment improved IS grain filling, IS grain weight 
a

Storage protein
Protein synthesis
Proteolysis
Protein folding

Fermentation

TCA cycle

Glycolysis

Glucose metabolism

Starch biosynthesis

b

Storage protein
Protein synthesis

Proteolysis

Protein folding

Fermentation

TCA cycle

Glycolysis

Glucose metabolism

Starch biosynthesis

C

Storage protein
Protein synthesis

Proteolysis

Protein folding

Fermentation
Glycolysis

Glucose metabolism

Starch biosynthesis

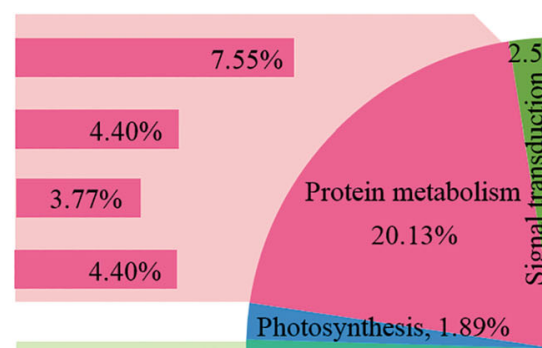

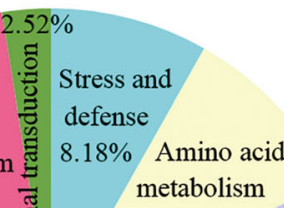

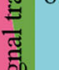
9.43\% Material $3.77 \%$ transport

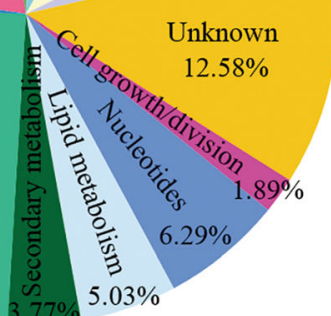

$9.43 \%$ $5.03 \%$
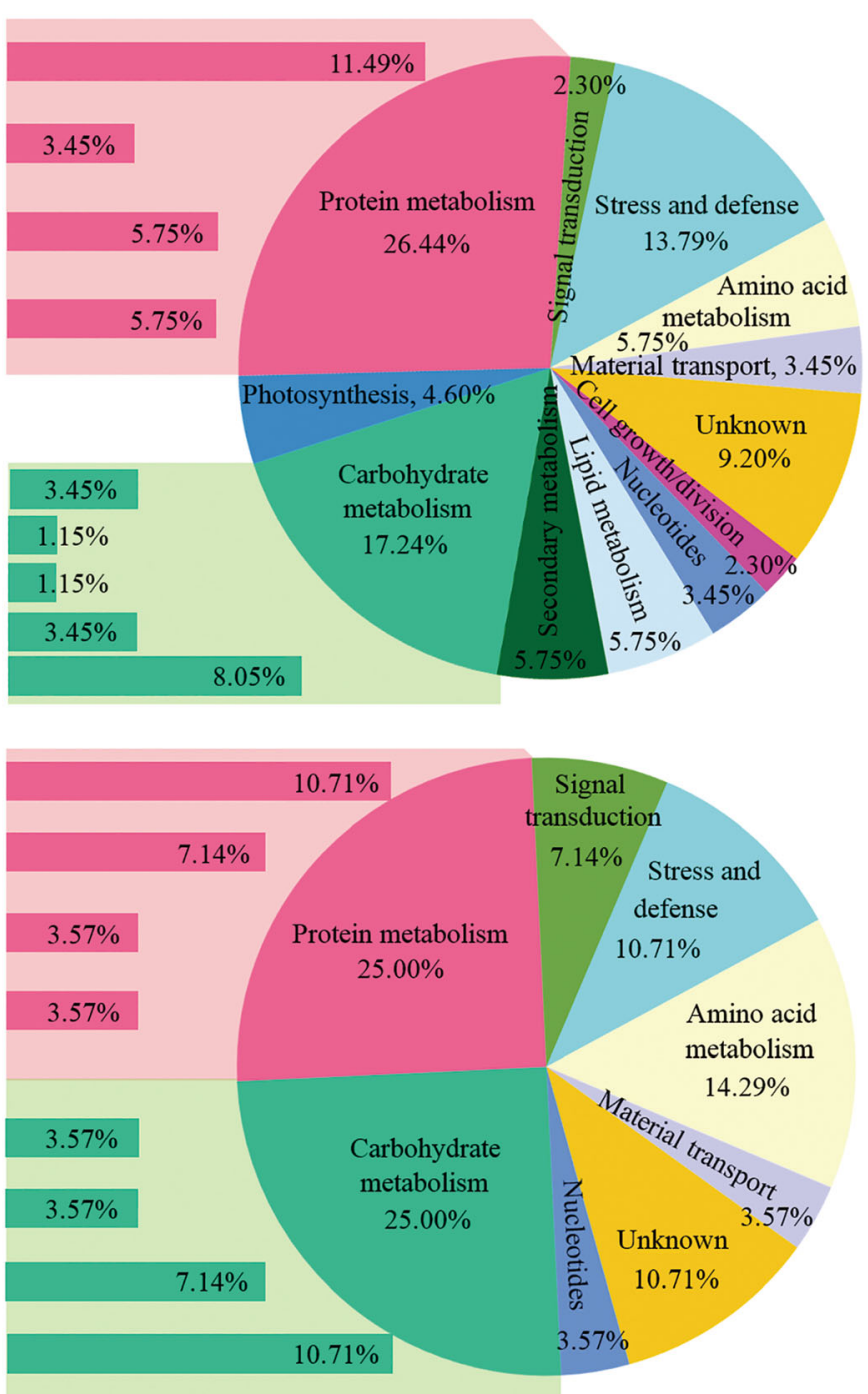

Fig. 4 Functional classifications of the differentially expressed proteins in groups a (TO-SS / TO-IS), b (TO-SS / T2-IS) and c (T2-IS / T0-IS). T0 represent control treatment with no spikelet thinning and T2 represent treatment with the upper 2/3 of spikelets were removed 
was still lower under T2 than that of T0-SS. Actin, which is involved in endosperm cell division, was 1.76-fold higher in T0-SS than in T2-IS, indicating that compared with T0-SS, sink capacity was smaller in T2-IS, thus explaining the low grain weight in T2IS at the protein expression level.

Amide-linked conjugates of indole-3-acetic acid (IAA) may serve as reservoirs of inactive IAA that can be hydrolyzed by IAA-conjugate hydrolases, releasing free IAA from the conjugate form. Thus, IAA-conjugate hydrolases are likely to play an important role in regulating free IAA levels $[37,38]$. For example, in maize germination, conjugate hydrolysis provides free IAA to the developing seedling [39]. IAA-amino acid hydrolase ILR1-like 8 is an IAA-conjugate hydrolase, and increasing its abundance could elevate levels of free IAA. IAA is an important signal in cereal endosperm development [40]. Low IAA leads to low endosperm cell division in rice IS [41]. Based on these findings, the expression of IAA-amino acid hydrolase ILR1-like 8 may be important for endosperm cell division. Our comparative proteomic results showed that the abundance of IAA-amino acid hydrolase ILR1-like 8 in T0-IS was 3.52-fold lower than that in T0-SS, suggesting that levels of free IAA in T0-IS were lower than those in T0-SS. Thus, T0-IS resulted in poor endosperm cell division, as well as low sink capacity and grain weight. While in T2-IS, the abundance of IAA-amino acid hydrolase ILR1-like 8 was still lower than in T0-SS, and the kernel development of T2-IS also poorer than that in T0-SS. These results indicate that the abundances of actin, annexins, and IAA-amino acid hydrolase ILR1-like 8 in rice are important for the establishment of grain sink.

\section{Low activities of key enzymes associated with sucrose- starch metabolism leading to poor starch synthesis} Grain filling is actually a process of starch biosynthesis and accumulation [42]. Grain filling materials are transported from the source to the grain mainly in the form of sucrose and are converted to starch through a series of enzymatically catalyzed reactions. Among these, sucrose synthase (SuSase) catalyzes and degrades sucrose to produce uridine diphosphoglucose (UDPG) and fructose, and its activity is an index of the rice sink strength [43]. In this study, the abundances of SuSase in SS were higher than those in IS at 10 DPA, which may be attributed to the high sucrose content of SS that needs to be decomposed. In the T2-IS/T0-IS and T0-SS/T2-IS comparisons, the abundance of SuSase was up-regulated and down-regulated, respectively, consistent with sucrose content. Studies have also shown that sucrose exerts a regulatory effect on SuSase activity [7, 44]. Under SS removal, a large amount of assimilate is supplied to the IS, increasing its sucrose content and inducing an increase in SuSase abundance. Therefore, the improvement in IS grain filling after the removal of SS may be attributed to increased assimilates and a stronger capacity for sugar decomposition.

Many enzymes involved in starch synthesis were identified in this study, such as ADP-glucose pyrophosphorylase (AGPase), starch branching enzyme (SBE), OSJNBa0019G23.2 protein (pullulanase), and putative starch synthase DULL1 (SSS). Among these, AGPase is a key enzyme controlling starch accumulation rate, and its up-regulation can achieve high yields [45]. SBE is a key enzyme controlling amylopectin synthesis, and its enzymatic activity is significantly positively correlated with the amylopectin accumulation rate [46], while SSS plays an important role in amylose synthesis [47]. In this study, compared to T0-IS at $10 \mathrm{DPA}$, the protein abundances of AGPase, SSS, SBE, and pullulanase in T0-SS were up-regulated. This result is consistent with the proteomic results from Zhang et al. [48]. Futhermore, compared to levels in T0-IS, alpha-glucosidase (AGS), which is involved in starch hydrolysis, was downregulated in T0-SS, while the alpha-amylase/subtilisin inhibitor (ASI), involved in the inhibition of starch hydrolysis, was up-regulated in T0-SS. This facilitated starch accumulation in SS. In general, the results from this study and from previous studies [49] show that reduced activity of the enzymes associated with starch synthesis is the main reason for poor IS grain filling.

As Fig. 5 shows, the DEPs related to carbohydrate metabolism in the T2-IS/T0-IS comparison mainly participate in starch synthesis. Compared to T0-IS, the abundances of SSS, SBE, and pullulanase in T2-IS were all up-regulated, which may be due to the increasing supply of sucrose to the IS after SS removal. Similar to the findings of previous studies, we showed that expressions of SSS and SBE were up-regulated by an increase in sucrose [50]. Therefore, improving IS grain filling after SS removal may be achieved through an increase in the sucrose content, which in turn induces the upregulation of SBE and SSS, thus promoting starch synthesis in IS.

\section{Weakened photosynthesis and respiration resulting in stagnation of grain development}

Carbohydrate metabolism mainly includes glycolysis and the TCA cycle, which provides energy and material for the transformation and synthesis of metabolites [51]. In this study, proteins associated with carbohydrate metabolism were identified (Fig. 5), including proteins involved in glycolysis, such as fructose-bisphosphate aldolase (FBA), glyceraldehyde3-phosphate dehydrogenase 3 (GAPDH), lactoylglutathione lyase, and phosphoglycerate kinase (PGK), as well as proteins participating in the TCA cycle, such as 


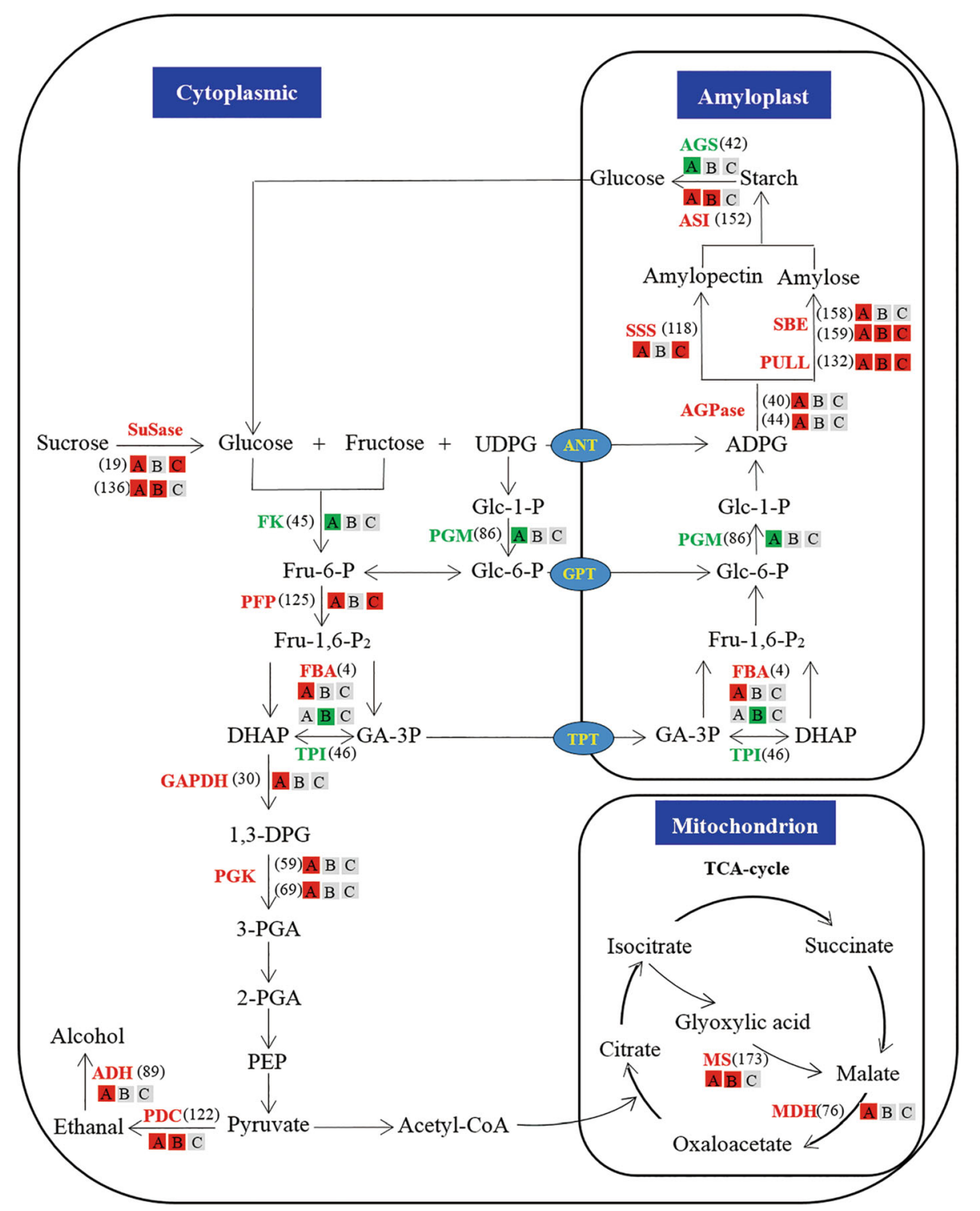

Fig. 5 The differentially expressed proteins onto carbohydrate metabolism of rice grain. Note: A, B and C represent the groups of T0-SS / TO-IS, TO-SS / T2-IS and T2-IS / TO-IS. Red and green indicate $p \leq 0.05$ (red denotes significant up-regulation in the endosperm, green significant down-regulation); Light grey indicate no significant difference in the level of $p \leq 0.05$. SuSase, sucrose synthase; SBE, starch branching enzyme; UDPG, uridine diphosphate glucose; ADPG, adenosine diphosphate glucose; DHAP, dihydroxyacetone phosphate; GA-3P, glyceraldehyde-3-phosphate; 1,3-DPG, 1, 3-diphosphoglycerate; 3-PGA, 3-phosphoglycerate; 2-PGA, 2-phosphoglycerate; PEP, phosphoenolpyruvate; FK, fructokinase; PFP, pyrophosphate-fructose 6-phosphate 1-phosphotransferase; PGM, phosphoglucomutase; AGPase, adenosine diphosphoglucose pyrophosphprylase; PULL, pullulanase; DULL1, putative starch synthase DULL1; FBA, fructose-bisphosphate aldolase; TPI, triosephosphate isomerase; AGS, alpha-glucosidase; ASI, alpha-amylase/subtilisin inhibitor; GAPDH, glyceraldehyde-3-phosphate dehydrogenase; PGK, phosphoglycerate kinase; PDC, pyruvate decarboxylase; ADH, alcohol dehydrogenase; MS, malate synthase; MDH, malate dehydrogenase, cytoplasmic; ANT, adenylate transporter; GPT, glucose phosphate translocator; TPT, triose phosphate translocator

malate dehydrogenase (MDH). The abundances of these proteins were lower in IS than in SS, which is consistent with the results of Zhang et al. [48]. Reduced glycolysis and TCA cycle activity in the IS at 10 DPA is not able to supply enough material and energy for cell expansion and starch synthesis, and thus affects the formation of the grain sink. Under hypoxic conditions, enzymes in the alcohol fermentation pathway are important for the formation of ATP, which is required to maintain starch synthesis, including pyruvate decarboxylase 2 (PDC 2) and alcohol dehydrogenase 2 (ADH2). The abundances of these two enzymes in the IS were lower than those in the SS, and this indicated that the down-regulation of alcohol 
fermentation in the IS resulted in a decrease in ATP, thus affecting the initiation of IS grain filling and restricting normal starch synthesis.

We also identified other proteins associated with energy metabolism, such as D-2-hydroxyglutarate dehydrogenase (D-2HGDH), which catalyzes the formation of 2-ketoglutarate from D-2-hydroxyglutarate in the mitochondria and releases energy [52], and formate dehydrogenase (FDH), which catalyzes the oxidation of formic acid to $\mathrm{CO}_{2}$ and reduces $\mathrm{NAD}^{+}$to NADH $[53,54]$. Our proteomic study indicated that these two proteins were down-regulated in the IS at 10 DPA. Ribulose bisphosphate carboxylase large chain (Rubisco), a key enzyme for $\mathrm{CO}_{2}$ fixation during plant photosynthesis [55], was identified in the present study, and its abundance in SS was 2.74-fold higher than that in IS. It may be inferred therefore that photosynthesis was more productive in SS than in IS, producing more carbohydrates used for grain filling. The reduced abundances of these energy metabolism-related proteins in the IS therefore explains poor IS grain filling at the proteomic level.

Pyrophosphate-fructose 6-phosphate 1phosphotransferase (PFP) can reversibly catalyze the conversion between fructose-6-phosphate (F6P) and fructose 1,6-bisphosphate (F-1,6- $\left.\mathrm{P}_{2}\right)$ by phosphorylation and dephosphorylation [56]. However, in vivo, the positive reaction from F6P to $\mathrm{F}-1,6-\mathrm{P}_{2}$ is catalyzed by the irreversible enzyme phosphofructokinase (PFK). It is worth noting that, unlike PFK, the positive reaction catalyzed by PFP does not require consumption of ATP. Therefore, in higher plants, oxygen-free glycolysis is dependent on PFP, which is more economical from the standpoint of energy transformation. PFP also stores energy in a PPi (phosphate group) from the perspective of gluconeogenesis [57]. In this study, we found that the PFP abundance of T2-IS was 2.27-fold higher than that of T0-IS, which demonstrated that PFP plays an important role in the processes of glycolysis and gluconeogenesis in IS after SS removal and that its activity is conducive to the economical utilization of energy.

\section{Low abundances of proteins associated with proteins metabolism (protein synthesis, folding, and storage) leading to poor protein synthesis}

Rice protein formation is closely related to the nitrogen nutritional status of the plant [58], which is regulated by nitrogen metabolism. Transamination is a crucial process of nitrogen metabolism, that involves a variety of enzymes, including aspartate amino transferase (GOT) and alanine amino transferase (GPT). In higher plants, inorganic nitrogen is converted to amino acids by catalysis with these two transaminases, thus providing a variety of amino acid donors for the synthesis and metabolism of grain proteins [59]. In this study, the abundances of GOT and GPT in SS were 3.82-fold and 3.07-fold higher, respectively, than those in IS, which may be due to the fact that in the early filling stage, less material was supplied to the IS, resulting in poor nitrogen metabolism. A proteomic study by Zhang et al. [48] has shown that the abundances of GPT in IS are downregulated, probably owing to the lack of nitrogen and accelerated aging of the rice plants at later stages. The results also showed that the abundances of GOT and GPT in T2-IS were higher than those in T0-IS, which suggested that IS grain filling was improved after SS removal, probably due to the up-regulation of GPT and GOT, promoting IS protein formation.

Molecular chaperones are effective in regulating the proper folding of polypeptide chains, thereby forming active proteins [60]. In this study, molecular chaperones, such as the DnaK-type molecular chaperone Bip and the chaperone protein ClpB1, were found to be differentially expressed between SS and IS. The abundances of these two proteins in T0-SS were 6.30-fold and 4.54-fold higher than those in T0-IS, while they were all higher in T2-IS than those in T0-IS. Thus, well-developed rice grains probably require high abundances of molecular chaperones, which regulate the proper folding of polypeptide chains.

The formation or isomerism of disulfide bonds plays an important role in protein folding and metabolic regulation [61]. Protein disulfide isomerase (PDI) and protein disulfide isomerase-like (PDILs) can catalyze the formation of disulfide bonds in proteins [62]. Shimoni et al. [63] was the first to report that PDI was involved in the folding of storage proteins during endosperm formation. In this study, the abundances of PDIL1-1, PDIL1-4, and PDIL2-3 in SS were all higher than those in IS. Johnson et al. [64] demonstrated that in wheat, PDIL1-1 was essential for accurate assembly and distribution of gliadin and glutelin in the endoplasmic reticulum. Moreover, PDIL1-1 was found to control endosperm development by regulating the quantity and composition of proteins in rice seeds [65]. The results of this study indicated that the synthesis of storage proteins in SS is elevated compared to that in IS during the formation of the seed endosperm, and this may be one of the reasons for poor IS grain filling. Additionally, the abundance of PDIL1-4 in T2-IS was higher than that in T0-IS, which may be attributed to a significant increase in the nitrogen compounds supplied to IS after SS removal. The quantity of storage proteins in IS was increased by upregulating PDIL abundance during the formation of the seed endosperm, thereby improving IS grain filling.

Storage proteins are mainly found in the rice endosperm and can be divided into glutelin, globulin, albumin, and prolamin according to their solubility. The contents and proportions of these proteins affect the 
quality of rice. In this study, we identified a large number of differentially expressed storage proteins between SS and IS, such as glutelin, globulin, and vegetative storage proteins (cupin family protein). The abundances of glutelin (Nos. 145, 157, 169) were 9.51-fold, 11.87-fold, and 14.62-fold higher in T2-IS than those in T0-IS, respectively. Ma et al. [66] showed that hightemperature stress significantly increased glutelin abundance in rice grains, but there was no effect of the application of panicle fertilizer. Dong et al. [22] suggested that drought stress may change the abundances of storage proteins in rice grains. The results of this study showed that during grain filling, SS removal could also affect the abundance of glutelin in the IS, though the specific regulatory mechanism involved requires further study.

\section{GTP binding protein, PP2C and IAA-amino acid hydrolase} ILR1-like 8, in signaling networks involved in IS development The growth and development of plants are mainly regulated by genetic and environmental information. The transmission of changing environmental information, namely, cellular signal transduction, regulates carbohydrate and energy metabolisms, as well as physiological and biochemical reactions. GTP binding protein participates in a series of signal transduction process in cells, such as the signal transduction of transmembrane messengers, light signal transduction, protein biosynthesis, and cytoskeletal structure formation [67]. In this study, the abundance of GTP-binding protein was 35.53 -fold higher in T0-SS than that in T0-IS, indicating that the rate of signal transduction in T0-SS was higher than that in T0-IS. In addition, the abundance of GTP-binding protein in T2-IS was also increased, and it was 12.89-fold higher compared to that in T0-IS, which may be one of the reasons for the improvement in IS grain filling after SS removal.

Protein phosphorylation/dephosphorylation is one of the most important methods of biological signal transmission, and it occurs mainly through the activities of two types of protein with mutually antagonistic biochemical properties: protein kinases and protein phosphatases. Protein phosphatase $2 \mathrm{C}$ (PP2C) plays an important role in biological signal transduction and is involved in various ABA signaling pathways in higher plants [68]. ABA is a key hormone involved in the regulation of grain filling, and ABA levels are significantly positively correlated with the grain filling rate [4]. In this study, the abundance of PP2C was 5.05-fold higher in SS than that in IS. Therefore, we suspect that poor IS grain filling may be associated with poor ABA signal transduction. The increase in IAA-amino acid hydrolase ILR1like 8 abundance may increase the active IAA level in grains. Seth et al. [69] argued that IAA as a signaling substance could control grain growth by regulating the distribution of assimilation products. The main role of IAA in grain filling is to increase the "pull" of its position to assimilates, so that assimilates are supplied primarily to locations with high IAA levels [70]. In this study, the abundance of IAA-amino acid hydrolase ILR1-like 8 in T0-SS was higher than that in T0-IS, suggesting that assimilates were preferentially supplied to the SS and that IS are unable to obtain a timely supply of nutrients after fertilization, resulting in a relative lag in IS grain filling.

\section{Increased abundance of 14-3-3 protein in IS inhibits starch synthesis}

In the process of plant development, 14-3-3 proteins participate in plant signal transduction, substance metabolism, stress response, and other regulatory processes by interacting with other proteins [71]. In recent years, great progress has been made in the study of plant 14$3-3$, and it was found that 14-3-3 also plays an important role in starch metabolism. A high content of 14-3-3 proteins in the wheat endosperm inhibits the activity of sucrose synthase [72]. In Arabidopsis, inhibition of 143-3 activity leads to an increase in starch accumulation [73]. These results suggest that 14-3-3 may inhibit starch synthesis. In this study, the abundance of 14-3-3 protein (14-3-3-like protein GF14-F) in IS was higher than that in SS, consistent with the results from previous studies [48]. Therefore, the higher abundance of 14-3-3 protein in IS could be an important factor leading to the poor development of IS.

\section{Unknown proteins}

Through bioinformatics comparison, we identified many unknown proteins, such as Nos. 83, 106, 156, and 168, which were over 3-fold up-regulated in T0-SS/T0-IS and T0-SS/T2-IS at 10 DPA. However, even using bioinformatics analysis methods, the roles of these proteins could not be identified, and their functions remain unclear.

\section{Conclusions}

A large-panicle japonica rice line W1844 was suitable to explore the physiological and molecular mechanism of poor IS grain filling, for there exists great difference in kernel development between SS and IS. Compared with SS, the IS exhibited more weaker endosperm cell division and lower activity of key enzymes related to sucrose-starch metabolism, carbohydrate metabolism and nitrogen metabolism. In addition, the weakened photosynthesis and respiration could not timely provide enough materials and energy for cell expansion and grain filling, which may result in the stagnation of IS development. Moreover, a higher abundance of 14-3-3 protein in IS could be involved in the inhibition of 
starch synthesis. However, the removal of SS significantly improved IS grain filling primarily by increasing carbohydrate supply, which increased the activities of key enzymes involved in sucrose-to-starch metabolism and nitrogen metabolism, promoting the starch and protein synthesis. Additionally, the energy metabolism was also improved as the more carbohydrate in IS. Therefore, we argued that a limitation in the assimilate supply may be the main cause of poor IS grain filling. The poor IS grain filling is a complex process and this is confirmed by the proteomic analysis in present study. An integrated method using a combination of omics platforms such as metabolomic and transcriptomic will be needed to understand this mechanism comprehensively.

\section{Additional file}

Additional file 1: List of all proteins identified and quantified by UPLC/ MS/MS. (XLSX $59 \mathrm{~kb})$

\section{Abbreviations}

2-DE: two-dimensional gel electrophoresis; ABA: Abscisic acid ADPG: Adenosine diphosphate glucose; AGPase: ADP-glucose pyrophosphorylase; DEPs: Differentially expressed proteins; DPA: Days post anthesis; GOT: Aspartate amino transferase; GPT: Alanine amino transferase; IAA: Indole-3-acetic acid; IS: Inferior spikelets; ITRAQ: Isobaric tags for relative and absolute quantitation; MS: Medium spikelets; PFP: Pyrophosphate-fructose 6-phosphate 1-phosphotransferase; PULL: Pullulanase; SBE: Starch branching enzyme; SS: Superior spikelets; SSS: Soluble starch synthase; SuSase: Sucrose synthase; TCA: Tricarboxylic acid; UDPG: Uridine diphosphoglucose

\section{Acknowledgements}

We thank the staff of the Laboratory of Crop Physiology and Ecology in Anhui Agriculture University and the Key Laboratory of Crop Physiology and Ecology in Southern China, Nanjing Agriculture University.

\section{Funding}

This work was supported by grants from the National Key Research and Development Program (2016YFD0300505; 2016YFD0300608) and Anhui Scientific and Technological Research Plan (1501031088).

\section{Availability of data and materials}

The data sets supporting the conclusions of this article are included within the article and its additional files. The data of matched proteins in Additional file 1 are from UniProt database. (http://www.uniprot.org/uniprot/).

\section{Authors' contributions}

$C Y, L W$ and SW designed the experiments; $L C$ and $H H$ performed part of the experiments; $C Y$ and $Y D$ analyzed experimental results; $C Y, L W, S W$ and $C M$ prepared the manuscript. All authors have read and approved the final manuscript.

\section{Competing interests}

All the authors declare that they have no conflict of interest.

\section{Consent for publication}

Not applicable.

\section{Ethics approval and consent to participate} Not applicable.

\section{Publisher's Note}

Springer Nature remains neutral with regard to jurisdictional claims in published maps and institutional affiliations.

\section{Author details}

'College of Agronomy, Anhui Agricultural University, Hefei 230036, People's Republic of China. ${ }^{2}$ College of Agronomy, Nanjing Agricultural University/Key Laboratory of Crop Physiology Ecology and Production Management, Ministry of Agriculture, Nanjing 210095, People's Republic of China. ${ }^{3}$ Jiangsu Collaborative Innovation Center for Modern Crop Production, Nanjing 210095, People's Republic of China.

Received: 12 January 2017 Accepted: 29 May 2017

Published online: 07 June 2017

\section{References}

1. Khush GS. What it will take to feed 50 billion rice consumers in 2030. Plant Mol Biol. 2005;59:1-6.

2. Kato T, Shinmura D, Taniguchi A. Activities of enzymes for sucrose-starch conversion in developing endosperm of rice and their association with grain filling in extra-heavy panicle types. Plant Prod Sci. 2007;10:442-50.

3. Yang J, Zhang J. Grain filling problem in "super" rice. J exp Bot. 2010;61:1-5.

4. Zhang H, Tan G, Yang L, Yang J, Zhang J, Zhao B. Hormones in the grains and roots in relation to post-anthesis development of inferior and superior spikelets in japonica/indica hybrid rice. Plant Physiol Biochem. 2009;47:195-204.

5. Ishimaru T, Matsuda T, Ohsugi R, Yamagishi T. Morphological development of rice caryopses located at the different positions in a panicle from early to middle stage of grain filling. Funct Plant Biol. 2003;30:1139-49.

6. Mohapatra PK, Patel R, Sahu SK. Time of flowering affects grain quality and spikelet partitioning within the rice panicle. Aust J Plant Physiol. 1993; 1993(20):231-42.

7. Tang $T$, Hong $X$, Yu X, Bing L, Jian $S$. The effect of sucrose and abscisic acid interaction on sucrose synthase and its relationship to grain filling of rice (Oryza sativa L.). J exp Bot. 2009;60(9):2641-52.

8. Wang Z, Xu Y, Chen T, Zhang H, Yang J, Zhang J. Abscisic acid and the key enzymes and genes in sucrose-to-starch conversion in rice spikelets in response to soil drying during grain filling. Planta. 2015;241(5):1091-107.

9. Fu J, Huang Z, Wang Z, Yang J, Zhang J. Pre-anthesis non-structural carbohydrate reserve in the stem enhances the sink strength of inferior spikelets during grain filling of rice. Field Crops res. 2011;123:170-82.

10. Peng T, Lv Q, Zhang J, Li JZ, Du YX, Zhao QZ. Differential expression of the microRNAs in superior and inferior spikelets in rice (Oryza sativa). J exp Bot. 2011;62(14):4943-54.

11. Ishimaru T, Hirose T, Matsuda T, Goto A, Takahashi K, Sasaki H, et al. Expression patterns of genes encoding carbohydrate-metabolizing enzymes and their relationship to grain filling in Rice (Oryza sativa L.): comparison of caryopses located at different positions in a panicle. Plant Cell Physiol. 2005; 46(4):620-8.

12. Xu FX, Xiong H, Zhu YC, Wang GX. Effect of source-sink ratio on grain filling and the source-sink characteristics of high yield varieties of mid-season hybrid rice. Scientia Agric sin. 2005;38(2):265-71.

13. Seo SW. The effects of the limiting of the number of the ripening grains per panicle on the changing of the grain weight and the protein content in brown rice during the ripening period. Jpn J Crop Sci. 1980;49:8-14.

14. Xu FX, Guo XY, Zhang L, Xiong H, Zhu YC, Liu M, et al. Effects of sink-source structures on filling of superior and inferior spikelets of mid-season hybrid rice. J Agr Sci Tech. 2013;15(1):96-101.

15. Kato $T$. Effect of spikelet removal on the grain filling of Akenohoshi, a rice cultivar with numerous spikelets in a panicle. J Agric Sci. 2004;142:177-81.

16. You CC, Zhu HL, Xu BB, Huang WX, Wang SH, Ding YF, et al. Effect of removing superior Spikelets on grain filling of inferior Spikelets in Rice. Front Plant Sci. 2016;7:1161.

17. Zieske $L R$. A perspective on the use of $\mathrm{iTRAQ}{ }^{\mathrm{TM}}$ reagent technology for protein complex and profiling studies. J exp Bot. 2006;57(7):1501-8.

18. Zhang ZX, Chen J, Lin SS, Li Z, Cheng RH, Fang CX, et al. Proteomic and phosphoproteomic determination of ABA's effects on grain-filling of Oryza sativa L. Inferior spikelets. Plant Sci. 2012;185-186:259-73.

19. Zhang ZX, Tang J, Du TW, Zhao H, Li Z, Li Z, et al. Mechanism of developmental stagnancy of rice inferior spikelets at early grain-filling stage as revealed by proteomic analysis. Plant Mol Biol rep. 2015;33(6):1844-63.

20. Chen $\Pi$, Xu GW, Wang ZQ, Zhang H, Yang JC, Zhang JH. Expression of proteins in superior and inferior spikelets of rice during grain filling under different irrigation regimes. Proteomics. 2015;16:102-21. 
21. Robbins ML, Roy A, Wang PH, Gaffoor I, Sekhon RS, de O. Buanafina MM, et al. Comparative proteomics analysis by DIGE and iTRAQ provides insight into the regulation of phenylpropanoids in maize. J Proteome. 2013;20(93):254-75.

22. Dong MH, Gu JR, Zhang L, Chen PF, Liu TF, Deng JH, et al. Comparative proteomics analysis of superior and inferior spikelets in hybrid rice during grain filling and response of inferior spikelets to drought stress using isobaric tags for relative and absolute quantification. J Proteome. 2014;109:382-99.

23. Richards F. A flexible growth function for empirical use. J exp Bot. 1959; 10(29):290-300.

24. Isaacson T, Damasceno CM, Saravanan RS, He Y, Catalá C, Saladié M, et al. Sample extraction techniques for enhanced proteomic analysis of plant tissues. Nat Protoc. 2006;1(2):769-74.

25. Smith PK, Krohn RI, Hermanson GT, Mallia AK, Gartner FH, Provenzano $\mathrm{MD}$, et al. Measurement of protein using bicinchoninic acid. Anal Biochem. 1985;150(1):76-85

26. Wiśniewski JR, Zougman A, Nagaraj N, Mann M. Universal sample preparation method for proteome analysis. Nat Methods. 2009;6:359-62.

27. Shilov IV, Seymour SL, Patel AA, Loboda A, Tang WH, Keating SP, et al. The paragon algorithm, a next generation search engine that uses sequence temperature values and feature probabilities to identify peptides from tandem mass spectra. Mol Cell Proteomics. 2007;6(9): 1638-55.

28. Kobata T, Akiyama Y, Kawaoka T. Convenient estimation of unfertilized grains in rice. Plant Production Sci. 2010;13(3):289-96.

29. Yang JC, Zhang WH, Wang ZQ, Liu LJ, Zhu QS. Source-sink characteristics and the translocation of assimilates in new plant type and intersubspecific hybrid rice. J Integr Agr. 2002b;1(2):155-62.

30. Xie GH, Yang JC, Wang ZQ, Zhu QS. Grain filling characteristics of rice and their relationships to physiological activities of grains. Acta Agron sin. 2001;27(5):557-65

31. Yang J, Zhang J. Grain filling of cereals under soil drying. New Phytol. 2006; 169:223-36.

32. Yang JC, Zhang JH, Huang Z, Wang ZQ, Zhu QS, Liu L. Correlation of cytokinin levels in the endosperms and roots with cell number and cell division activity during endosperm development in rice. Ann Bot. 2002a; 90:369-77.

33. Singh BK, Jenner CF. Association between concentration of organic nutrients in the grain, endosperm cell number and grain dry weight within the ear of wheat. Aust J Plant Physiol. 1982;9(1):83-95.

34. Jones R, Roessler J, Ouattar S. Thermal environment during endosperm cell division in maize: effects on number of endosperm cells and starch granules. Crop Sci. 1985;25:830-4.

35. Hall A. Rho GTPases and the Actin cytoskeleton. Science. 1998;279(5350): 509-14.

36. Emoto K, Sawada H, Yamada Y, Fujimoto H, Takahama Y, Ueno M, et al. Annexin II overexpression is correlated with poor prognosis in human gastric carcinoma. Anticancer res. 2001;21(2B):1339-45.

37. Bartel B, Fink GR. ILR1, an amidohydrolase that releases active indole-3acetic acid from conjugates. Science. 1995;268:1745-8.

38. Ludwig-Müller J, Epstein E, Hilgenberg W. Auxinconjugate hydrolysis in Chinese cabbage: characterization of an amidohydrolase and its role during infection with clubroot disease. Physiol Plant. 1996;96:627-34.

39. Epstein E, Cohen JD, Bandurski RS. Concentration and metabolic turnover of indoles in germinating kernels of Zea mays L. Plant Physiol. 1980;65:415-21.

40. Lur HS, Setter TL. Role of auxin in maize endosperm development (timing of nuclear DNA endoreduplication, zein expression, and cytokinin). Plant Physiol. 1993;103:273-80.

41. Yang JC, Zhang JH, Wang ZQ, Zhu QS. Hormones in the grains in relation to sink strength and postanthesis development of spikelets in rice. Plant Growth Regul. 2003;41:185-95.

42. Yoshida S. Physiological aspects of grain yield. Annu rev Plant Physiol. 1972; 23:437-64.

43. Liang J, Zhang J, Cao X. Grain sink strength may be related to the poor grain filling of indica-japonica (Oryza sativa) hybrids. Physiol Plant. 2001;112: 470-7.

44. Ross HA, Davies HV. Sucrose metabolism in tubers of potato (Solanum tuberosum L.). Effects of sink removal and sucrose flux on sucrosedegrading enzyme. Plant Physiol. 1992;98:287-93.
45. Kang GZ, Liu GQ, Peng XQ, Wei L, Wang CY, Zhu YJ, et al. Increasing the starch content and grain weight of common wheat by over-expression of the cytosolic AGPase large subunit gene. Plant Physiol Biochem. 2013;73:93-8.

46. Mizuno K, Kimura K, Arai Y, Kawasaki T, Shimada H, Baba T. Starch branching enzymes from immature rice seeds. Biochem J. 1992;112(5):643-51.

47. Keeling $P$, Bacon $P$, Holt D. Elevated temperature reduces starch deposition in wheat endosperm by reducing the activity of soluble starch synthase. Planta. 1993;191:342-8.

48. Zhang ZX, Zhao H, Tang J, Li Z, Li Z, Chen DM, et al. A proteomic study on molecular mechanism of poor grain-filling of rice (Oryza sativa L.) inferior spikelets. PLoS One. 2014;9(2):1-19.

49. Fu J, Xu YJ, Chen L, Yuan LM, Wang ZQ, Yang JC. Changes in enzyme activities involved in starch synthesis and hormone concentrations in superior and inferior spikelets and their association with grain filling of super rice. Rice Sci. 2013;20:120-8.

50. Ahn YO, Kim SH, Kim CY, Lee JS, Kwak SS, Lee HS. Exogenous sucrose utilization and starch biosynthesis among sweet potato cultivars. Carbohydr res. 2010;345(1):55-60.

51. Fernie AR, Carrari F, Sweetlove LJ. Respiratory metabolism: glycolysis, the TCA cycle and mitochondrial electron transport. Curr Opin Plant Biol. 2004;7:254-61.

52. Achouri Y, Noël G, Vertommen D, Rider MH, Veiga-da-cunha M, Schaftingen EV. Identification of a dehydrogenase acting on D-2-hydroxyglutarate. Biochem J. 2004;381(1):35-42.

53. Bykova NV, Stensballe A, Egsgaard H, Jensen ON, Moller IM. Phosphorylation of formate dehydrogenase in potato tuber mitochondria. J Biol Chem. 2003; 278(28):26021-30.

54. Andreadeli A, Flemetakis E, Axarli I, Dimou M, Udvardi MK, Katinakis P, et al. Cloning and characterization of Lotus Japonicus formate dehydrogenase: a possible correlation with hypoxia. Biochim Biophys Acta. 2009;1794(6):976-84.

55. Lundqvist T, Schneider G. Crystal structure of activated ribulose-1,5bisphosphate carboxylase complexed with its substrate, ribulose-1,5bisphosphate*. J Biol Chem. 1991;266:12604-11.

56. Kowalczyk S, Januszewska B, Cymerska E, Maslowski P. The occurrence of inorganic pyrophosphate: D-fructose-6-phosphate 1-phosphotransferase in higher plants. Physiol Plant. 1984;60(1):31-7.

57. Botha AM, Botha FC. Effect of anoxia on the expression and molecular form of the pyrophosphate dependent phosphofructokinase. Plant Cell Physiol. 1991;32(8):1299-302.

58. Jiang LG, Dai TB, Wei SQ, Gan XQ, Xu JY, Cao WX. Genotypic differences and valuation in nitrogen uptake and utilization efficiency in rice. Acta Phytoecologica Sinica. 2003;27(4):466-71.

59. Zhao $X Q$, Shi WM. Expression analysis of the glutamine synthetase and glutamate synthase gene families in young rice (Oryza sativa) seedlings. Plant Sci. 2006;170:748-54.

60. Levinthal C. Are there pathways for protein folding? J Chim Phys. 1968;65:44-5.

61. Kaminaka H, Morita S, Yokoi H, Masumura T, Tanaka K. Molecular cloning and characterization of a cDNA for plastidic copper / zinc-superoxide dismutase in rice (Oryza sativa L.). Plant Cell Physiol. 1997;38(1):65-9.

62. Freedman RB, Hirst TR, Tuite MF. Protein disulphide isomerase : building bridges in protein folding. Trends Biochem Sci. 1994;19:331-6.

63. Shimoni Y, Segal G, Zhu XZ, Galili G. Nucleotide sequence of a wheat cDNA encoding protein disulfide isomerase. Plant Physiol 1995, 107 (1): 281-281.

64. Johnson JC, Appels R, Bhave M. The PDI genes of wheat and their syntenic relationship to the esp2 locus of rice. Funct Integr Genomic. 2006;6:104-21.

65. Kim YJ, Yeu SY, Park BS, Koh HJ, Song JT, Seo HS. Protein disulfide isomerase-like protein 1-1 controls endosperm development through regulation of the amount and composition of seed proteins in rice. PLOS One. 2012;7(9):e44493.

66. Ma QL, Li YS, Tian XH, Yan SZ, Lei WC, Noboru N. Influence of high temperature stress on composition and accumulation configuration of storage protein in rice. Sci Agric sin. 2009;42(2):714-8.

67. Yoshito K, Hiroshi I, Tohruk k, Masato N, Takaya S. Structure and function of signal-transducting GTP-binding proteins. Annu rev Biochem 1991, 60: 349-400.

68. Murata Y, Pei ZM, Mori IC, Schroeder J. Abscisic acid activation of plasma membrane $\mathrm{Ca}^{2+}$ channels in guard cells requires cytosolic $\mathrm{NAD}(\mathrm{P}) \mathrm{H}$ and is differentially disrupted upstream and downstream of reactive oxygen species production in abi1-1 and abi2-1 protein phosphatase $2 \mathrm{C}$ mutants. Plant Cell. 2001;13(11):2513-23. 
69. Seth A, Waering PE. Hormone-directed transport of metabolites and its possible role in plant senescence. J exp Bot. 1967;18:65-77.

70. Tian S, Wang X. The relationship between IAA and grain developing of Indica-japonica hybrid rice and regulation with S-07. Chinese J Rice Sci. 1998;12(2):99-104.

71. Aitken A. 14-3-3 pproteins: a historic overview. Semin Cancer Biol. 2006; 16(3):162-72.

72. Song JM, Dai S, Li HS, Liu AF, Cheng DG, Chu XS, et al. Expression of a wheat endosperm 14-3-3 protein and its interactions with starch biosynthetic enzymes in amyloplasts. Acta Agron sin. 2009;35(8):1445-50.

73. Sehnke PC, Chung HJ, Wu K, Ferl RJ. Regulation of starch accumulation by granule-associated plant 14-3-3 proteins. Proc Natl Acad Sci U SA. 2001;98: 765-70.

Submit your next manuscript to BioMed Central and we will help you at every step:

- We accept pre-submission inquiries

- Our selector tool helps you to find the most relevant journal

- We provide round the clock customer support

- Convenient online submission

- Thorough peer review

- Inclusion in PubMed and all major indexing services

- Maximum visibility for your research

Submit your manuscript at www.biomedcentral.com/submit 